\title{
Quantitative Analysis of Melanocyte Migration in vitro Based on Automated Cell Tracking under Phase Contrast Microscopy Considering the Combined Influence of Cell Division and Cell-Matrix Interactions
}

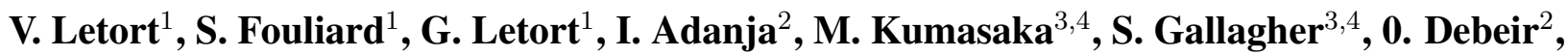 \\ L. Larue $^{3,4}$, and F. Xavier ${ }^{1 *}$ \\ ${ }^{1}$ Laboratory of Applied Mathematics, Ecole Centrale Paris, F-92295 Chatenay-Malabry, France \\ ${ }^{2}$ Laboratory of Image Synthesis and Analysis (LISA), Faculty of Applied Sciences, \\ Universite Libre de Bruxelles, 1050 Brussels, Belgium \\ ${ }^{3}$ Institut Curie, Centre Recherche, Developmental Genetics of Melanocytes, F-91405 Orsay, France \\ ${ }^{4}$ CNRS UMR146, F-91405 Orsay, France
}

\begin{abstract}
The aim of this study was to describe and analyze the regulation and spatio-temporal dynamics of melanocyte migration in vitro and its coupling to cell division and interaction with the matrix. The melanocyte lineage is particularly interesting because it is involved in both embryonic development and oncogenesis/metastasis (melanoma). Biological experiments were performed on two melanocyte cell lines established from wild-type and $\beta$-catenin-transgenic mice (bcat*). The multi-functional $\beta$-catenin molecule is known to be able to regulate the transcription of various genes involved in cell proliferation and migration, particularly in the melanocyte lineage. We also investigated fibronectin, an extra-cellular matrix protein that binds integrins, thereby providing adhesion points for cells and encouraging migration. As the migration of individual cells were followed, automated methods were required for processing the large amount of data generated by the time-lapse video-microscopy. A model-based approach for automated cell tracking was evaluated on a sample by comparison with manual tracking. This method was found reliable in studying overall cell behaviour. Its application to all the observed sequences provided insight into the factors affecting melanocyte migration in vitro: melanocytes of mutated form of $\beta$-catenin showed higher division rates and no contact inhibition of growth was induced by the resulting increase in cell density. However, cell density limited the amplitude of cell displacements, although their motility was less affected. The high fibronectin concentration bound to substratum promoted
\end{abstract}

\footnotetext{
*Corresponding author. E-mail: francoise.xavier@ecp.fr
} 
cell migration and motility, the effect being more intense for wild-type cells than for cells with $\beta$ catenin over-expression. During the division process, cell migration speed increased rapidly then decreased slowly. Analyses of such data is expected to lead both to biological answers and to a framework for a better modeling processes in the future.

Key words: melanocytes, automated cell tracking, video microscopy, cell migration, cell division, cell-matrix interaction, $\beta$-catenin, fibronectin.

AMS subject classification: $62 \mathrm{P} 10,92 \mathrm{C} 17$

\section{Introduction}

Life depends on survival and renewal, and both unicellular and multicellular organisms must find a non-toxic environment that provides them with the energy needed for interaction between cells and other organisms. The search for this suitable environment requires motility, which may be active, passive or symbiotic. In multicellular organisms, cell migration is a key process in embryonic development, homeostasis and cancer metastasis [2, 20]. Embryonic development and homeostasis are dynamic processes occurring over a predefined time period. For example, the gestation period in mice is 20 days and during this time many events occur that are subject to coordinated spatial and temporal regulation, such as cell division, determination, differentiation, cell death and migration. During development these cellular processes are coordinated by precise, interlinked molecular mechanisms. By contrast, during the formation of cancer and metastasis, these cellular and molecular processes occur in a non-coordinated manner.

The melanocyte lineage is a useful subject for studying the regulation and dynamics of these mechanisms and for determining how these mechanisms operate and interact during the establishment of the cell lineage, its renewal from the melanocyte stem cells and its transformation into an aggressive and devastating melanoma.

Melanocytes are derived from neural crest cells (NCC), considered by many to correspond to the fourth embryonic layer [33]. NCCs give rise to various cell types, which proliferate extensively and follow two main migration pathways: the dorso-ventral and dorso-lateral pathways. The founder melanoblasts proliferate actively to generate the precursor melanoblasts which migrate along the dorso-lateral pathway between the somites and the ectoderm. These precursors give rise to migrating melanoblasts that proliferate and migrate actively to the ventral part of the embryo. They cross the basement membrane separating the dermis from the epidermis and invade the epidermis while remaining in contact with the basement membrane [23]. In mice, most melanoblasts migrate to the growing hair follicle to produce the follicular melanoblasts, which differentiate into the mature melanocytes responsible for melanin production [21]. Melanocytes may undergo transformation to melanoma, sometimes after forming a naevus in which the classical heterotypic melanocyte-keratinocyte interaction is replaced by a homotypic melanocyte-melanocyte interaction. Cell-cell adhesion molecules are involved in melanoma formation and metastasis, and 
are affected by these processes. The molecules involved include those of the cadherin/catenin complex, which play a key role during normal and pathological development of melanocytes.

During the establishment of the melanocyte lineage, homeostasis and oncogenesis/metastasis, it becomes obvious that the migration of the melanocytes depends on their status in a given environment in a dynamic manner. In other words, cells migrate in cell autonomous (dependent on the energetic and cytoskeleton status of the cells for example) and in cell non-autonomous manners (which may involve cell-cell contact, cell-matrix contact and chemoattraction). Moreover, cells may be more or less prone to migrate depending for example on the cell cycle phase $\mathrm{G} 1 / \mathrm{S} / \mathrm{G} 2 / \mathrm{M}$, or specific local environmental conditions.

In this study, we carried out a quantitative analysis of melanocyte migration in vitro and investigated the effects of two important processes on cell migration: cell division and cell-matrix interaction. Individual cells were tracked using time lapse video-microscopy, either manually or using an automatic cell tracking algorithm [6], making it possible to screen large numbers of cells in different experimental conditions. This volume of data generated should compensate for interexperiment and inter-cellular variability inherent in biological studies. We focused in particular on the influence of two molecules: (i) $\beta$-catenin, a multi-functional protein that regulates the transcription of genes involved in cell proliferation and cell migration particularly [21], (ii) fibronectin (FN), an extracellular matrix glycoprotein that promotes cell migration in vitro, highlighting the link between extracellular matrix-mediated cell adhesion and migration [24]. Analyses of such data is expected to lead both to biological answers and to a framework for a better formalization and modeling processes in the future.

\section{Experimental background}

Mice producing an oncogenic form of $\beta$-catenin (bcat*) specifically in melanocytes were generated in a previous study and melanocyte cell lines from wild-type (WT) and bcat* mice were established and cultured as described previously [20,9].

For the time-lapse video microscopy analysis, duplicate wells of a 6-well plate were treated with 0,1 or $40 \mu \mathrm{g} / \mathrm{ml}$ fibronectin (Sigma, St Louis, Missouri) for $24 \mathrm{hr}$ at $4^{\circ} \mathrm{C}$. The coated dishes were then washed twice with PBS and blocked by incubating with $3 \% \mathrm{BSA} / \mathrm{PBS}(\mathrm{w} / \mathrm{v})$ for 1 $\mathrm{hr}$ at $37^{\circ} \mathrm{C}$ before the cells were seeded into the wells. Exponentially growing WT and bcat* melanocytes were dispensed into the plates at a density of $5 \times 10^{4}$ cells/well and incubated at $37^{\circ} \mathrm{C}$ with 5\% CO2 for 18hrs in F-12 media (Gibco, Carlsbad, California) supplemented with 10\% FBS and 200nM of the phorbol ester 12-O-tetradecanoylphorbol-13-acetate (TPA). After five hours starvation of FBS and TPA, time-lapse experiment was started. A digital image of the field of interest (size $647.3 \mu \mathrm{m} \times 483.6 \mu \mathrm{m}$ ) was taken every $4 \mathrm{~min}$ for 12 hours, with a scaling of $0.93 \mu \mathrm{m} / \mathrm{pixel}$, and the 180 images (or frames) generated were arranged into a sequence. The frame acquisition rate was chosen according to the observed typical cell displacement: with a 4-minutes period, the longest observed displacement is therefore limited to one or two cell diameter. Six fields were se- 
lected on each well (Figure 1). The whole experiment was performed three times, thus generating 19,440 frames in total $(180 \times 6 \times 6 \times 3)$, to ensure that the results obtained were statistically robust.

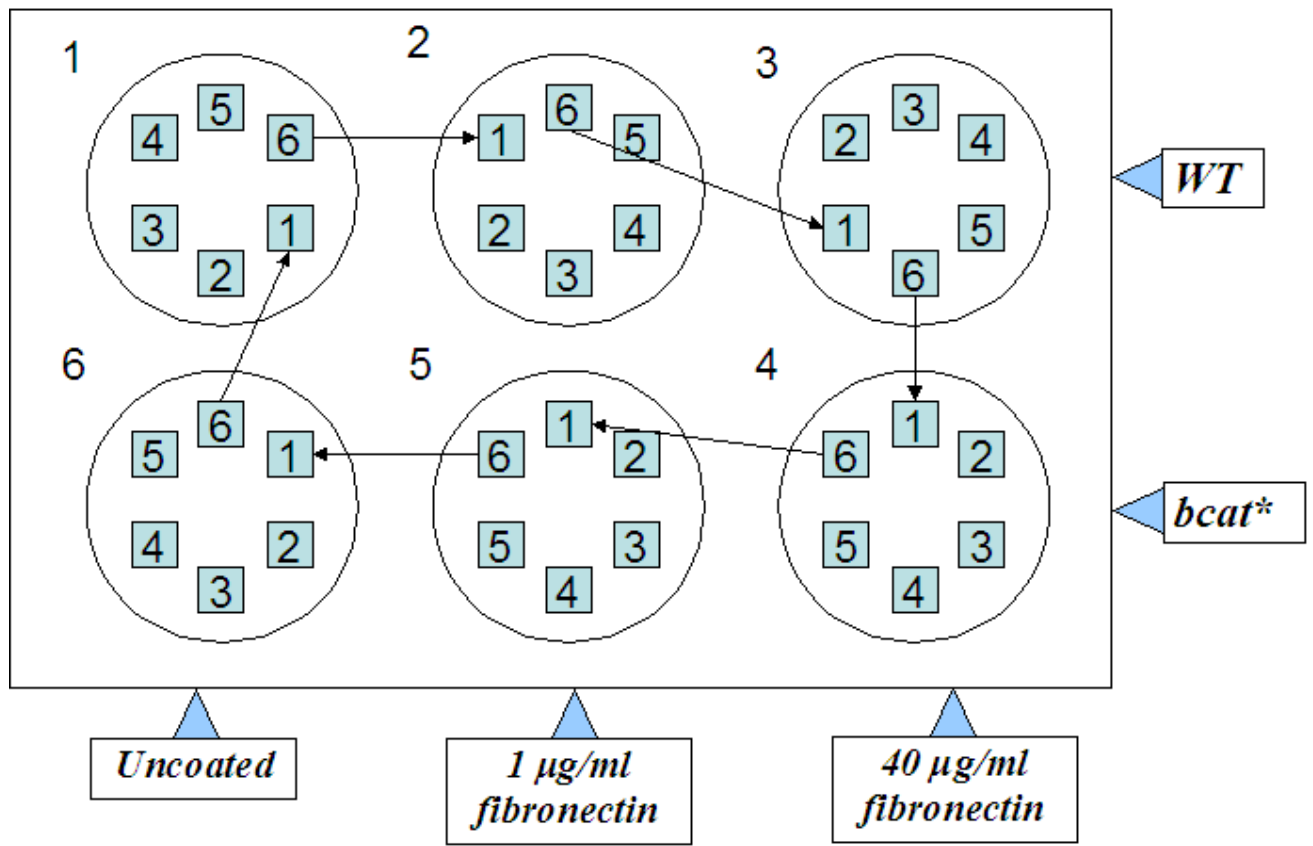

Figure 1: Schematic representation of the six photographic fields in each well of the microplate. Wells 1, 2 and 3 were seeded using Wild Type cells (WT), and wells 4, 5 and 6 were seeded using bcat* cells. Wells 1 and 6 were uncoated, wells 2 and 5 were coated with $1 \mu \mathrm{g} / \mathrm{ml}$ fibronectin, and wells 3 and 6 were coated with $40 \mu \mathrm{g} / \mathrm{ml}$ fibronectin. The arrows and the numbers represent the camera trajectory.

\section{Tracking and analysis of cell trajectories}

\subsection{Methods for in vitro cell tracking}

\subsubsection{Cell trajectory}

Manual and automatic methods were used to track melanocytes in vitro in time-lapse sequences. In the manual method, the operator had to click on every cell nucleus in each frame. The nucleus of each cell was followed from the first to the last frames and the results were recorded using the 'Manual Tracking' plug-in developed at Institut Curie by F. Cordelières for ImageJ (Version 1.41o, Wayne Rasband, National Institutes of Health, USA, http://rsb.info.nih.gov/ij). Each cell of the six photographed fields in each well-plate dish was analyzed for the three experiments. Due to the large amount of data (more than 200000 cell position clicks are required for each 6 well plate), manual tracking is a tedious task and therefore the automation of such a process is desirable. 
However, the tracking of unmarked cells (i.e. without fluorescent probe) in images acquired by a phase-contrast microscopy is a challenge for image-analysis software. In this context, cells are objects that display considerable variation in size and shape, can split due to cell division and can have incomplete or indistinct boundaries which may partially overlap.

Debeir et al. [7] have designed a method based on the mean-shift algorithm principle to overcome these difficulties and track cells in phase contrast images [4,6]. This algorithm iteratively converges from an initial point to the local density maximum of the underlying function. At each step, a 'kernel' is displaced from its current position to its center of mass. The image is interpreted as a discrete $2 \mathrm{D}$ function, the value of which is defined by the grey value of the corresponding pixel.

This algorithm thus converges to the brightest or the darkest zones of an image, depending on the weighting assigned to the grey values. In phase-contrast microscopy images, cells appear as dark objects surrounded by bright halos. Several triangle-shaped mean-shift kernels are grouped together in a concentric triangle-shaped pattern as illustrated in Figure 2 to cope with this cell pattern [7]. Inner smaller triangles are attracted by dark pixels (soma of the cell), wider triangles are attracted by bright pixels (refringent cell border). The new cell position is calculated by assembling the individual triangle positions.

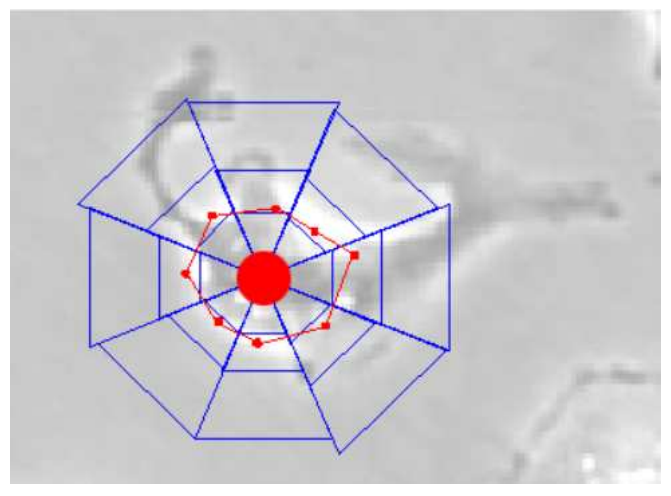

Figure 2: Phase contrast image of a cell with an ensemble of triangle shaped mean-shift kernels.

Cell division results in the production of two daughter cells and therefore adds new elements to the set of tracked objects. This problem of object splitting was dealt with by tracking cells backwards in time: divisions were therefore detected as fusions of two backward trajectories into one, that of the mother cell. The initialization step of the algorithm was performed on the last frame, which contained the largest number of cells: an operator manually identified the cells to be analyzed by clicking on cell nuclei positions. The trajectories of the cells were then extracted by reverse tracking.

According to the sensitivity analysis presented in a previous study [7], the following algorithm parameters must be fine-tuned. Thus, several trials showed an optimal tracking for 8 kernel pies, with a 14 threshold of the sigmoid function used in the model transition and a 1.2 factor for radial expansion of the model.

To estimate the reliability of automatic tracking, we applied both the manual and automatic 
methods to one experiment. It should be noted that, unlike automated tracking, manual tracking was carried out in the natural time direction (from the first to the last frame).

\subsubsection{Detection of division}

A custom division detector has been presented previously [8], but its use required interactive validation by an operator. In this study, divisions were detected manually, separately from the cell tracking process. The time of division was recorded along with the frame number at which two daughter cells could be first distinctly identified following a cell division.

Information relating to cell division can be extracted from phase-contrast time-lapse video microscopy (Figure 3).

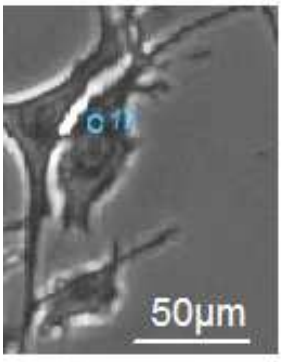

$-28 \mathrm{~min}$

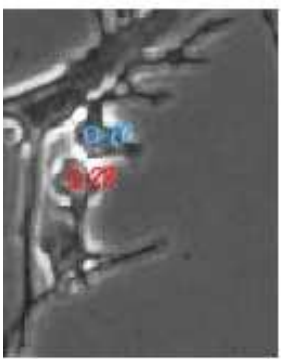

+8 min

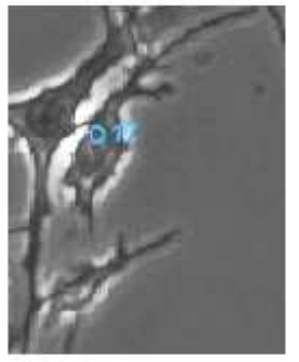

$-20 \min$

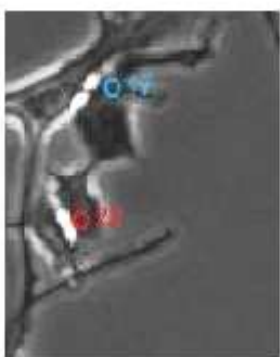

+24 min

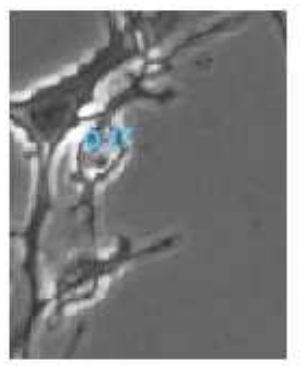

$-12 \min$

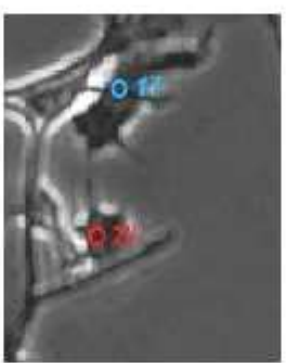

+36 min

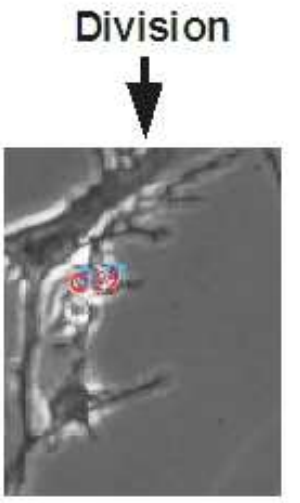

$0 \mathrm{~min}$

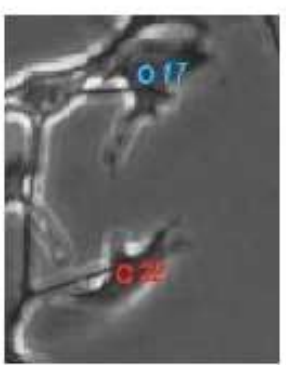

+64 min

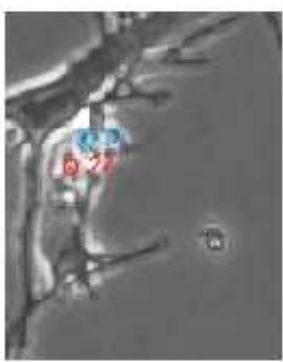

$+4 \min$

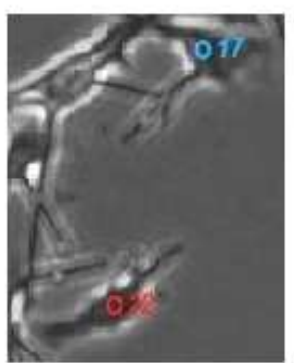

+104 min

Figure 3: Illustration by time-lapse image sequences showing cell events before and after the beginning of the division (time 0 ). The mother cell (number 17) is replaced by two other cells, one retaining the number 17 and the other taking the number 22 .

The number of cells dividing during the experiment was validated by a human operator to prevent some of the problems encountered with indirect tracking approaches (see [8]). This validation was rapid and straightforward, due to the characteristic roundness and brightness of the dividing cells (Figure 3). Before the division, the mother cell dramatically decreases its area and appears 
as a small bright disk. At the time of division, the daughter cells are spheres and appear to have similar volumes, but both cells are spreading and their surface area increases rapidly thereafter. The daughter cells then move away progressively.

\subsection{Criteria used to characterize cell migration}

Several indicators were defined to characterize cell trajectory and motility, that is cell migration. Although more elaborate indicators can be used, for instance when considering the trajectory as a persistent 2D random walk as done in [28], we chose here to simply define the trajectory of a cell as the set of its successive positions during a recorded sequence completed by linear interpolations to define the inter-frame paths. Instantaneous cell speed was defined as the cell displacement per unit of time between two successive frames, i.e. the euclidean distance between two successive cell positions divided by the time lapse (4min). If $x_{c}(t)$ and $y_{c}(t)$ are the coordinates of any cell $c$ at time $t$ (expressed as frame number), the instantaneous cell speed can be calculated as:

$$
s_{c}(t)=\frac{1}{4} \cdot \sqrt{\left(x_{c}(t-1)-x_{c}(t)\right)^{2}+\left(y_{c}(t-1)-y_{c}(t)\right)^{2}}
$$

Mean cell speed (MS) thus corresponds to the total distance covered during the experiment, divided by the duration of the experiment. MS was considered to be representative of cell motility.

The variability of instantaneous cell speed was assessed based on the sample standard deviation, $S D_{c}$, of the instantaneous speed sequence:

$$
S D_{c}=\frac{1}{N_{f}-1} \cdot \sum_{t=1}^{N_{f}}\left(s_{c}(t)-M S_{c}\right)^{2}
$$

where $N_{f}$ is the number of inter-frame intervals and $M S_{c}$ is the mean speed of cell $c$. The denominator $N_{f}-1$ was used, so that $S D_{c}$ provided an unbiased estimator of the population variance for independent and identically distributed observations (Bessel's corrections).

Two indicators were defined to characterize the cell trajectory: the trajectory amplitude and the final relative distance to origin.

We characterised the spatial extension of the cell trajectory by defining the trajectory amplitude (MaxDist) as the maximum distance between two positions of the sequence:

$$
\text { MaxDist }_{c}=\max \left\{\sqrt{\left(x_{c}\left(t_{1}\right)-x_{c}\left(t_{2}\right)\right)^{2}+\left(y_{c}\left(t_{1}\right)-y_{c}\left(t_{2}\right)\right)^{2}},\left(t_{1}, t_{2}\right) \in\left[t_{0}, t_{f}\right]^{2}\right\}
$$

where $t_{0}$ and $t_{f}$ are the initial and final frames of the experiment, respectively. This made it possible to distinguish migrating cells from cells displaying motility but stationary behaviour.

To assess the distance the cell migrated from its origin to the end of the observation (FRDO) we compute the linear distance between the initial and final cell position as the following equation:

$$
F R D O_{c}=\sqrt{\left(x_{c}\left(t_{f}\right)-x_{c}\left(t_{0}\right)\right)^{2}+\left(y_{c}\left(t_{f}\right)-y_{c}\left(t_{0}\right)\right)^{2}}
$$


with the same notations as in the previous equation. This feature allows to identify the statistical trend of cells that randomly explore a large area (i.e. go back in the neighbourhood of their initial position). This feature can help quantifying the classification of cell trajectories presented in [30]: for instance, palindromic cells, i.e. cells moving bi-directionally, would have high values for MaxDist but low values for FRDO. An example of this kind of trajectory is presented in Figure 4 along with annotations representing the features that were defined above. The 181 crosses mark the successive positions of the cell, which means that in zones of high density of crosses, the cell moved slowly while the low density of crosses seen at two portions of the tracking (frames 92-119 and 131-139, green-colored) indicate phases of rapid movement.

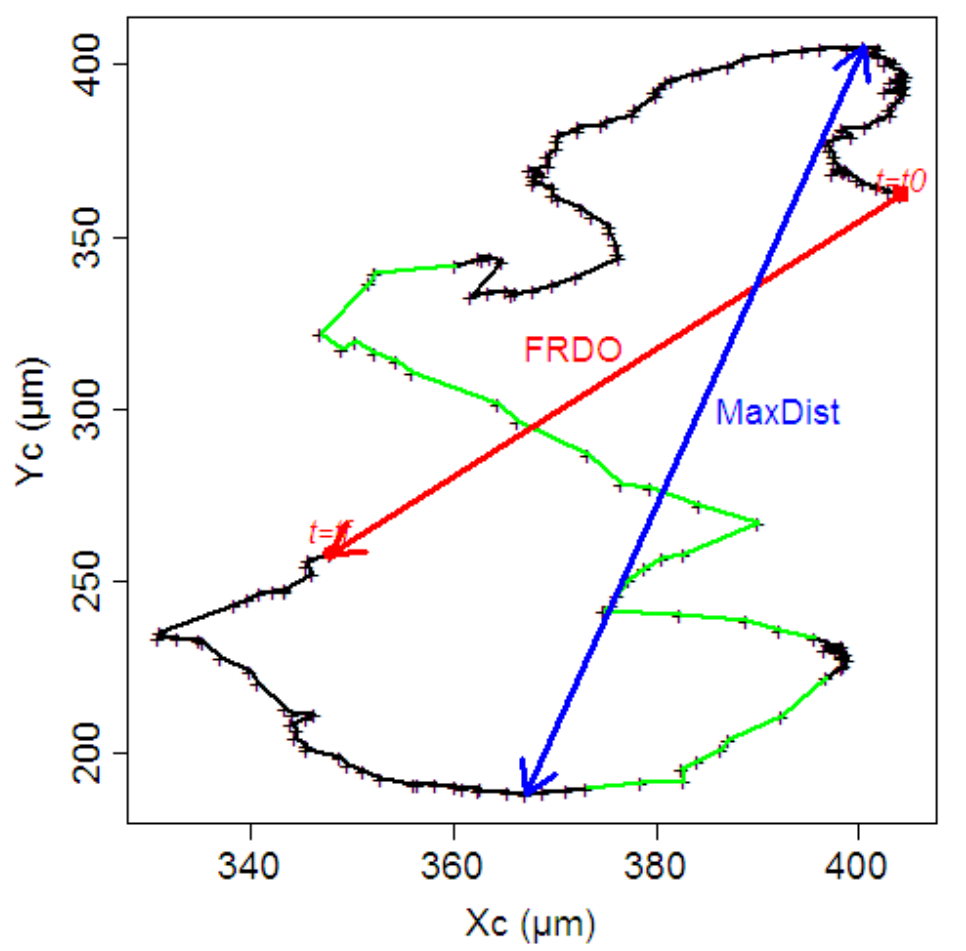

Figure 4: Diagram of two features defined to characterize cell trajectories: trajectory amplitude (MaxDist) and final relative distance to origin (FRDO). $t_{f}$ and $t_{0}$ mark respectively the initial and final position of the recorded sequence. Crosses (+) represent the successive positions of the cell at four-minute intervals. Mean speed (MS) is proportional to the total length of the black line. Green-colored portions indicate phase of rapid movement

\subsection{Statistical tests}

To assess the significance of the differences between two cell populations (grown in different conditions or from two different cell lines), several statistical tests were used in this paper. The null hypothesis was stated as that of equality of means in the two groups. Statistical tests thus give the 
probability of observing a value for the test statistic that is at least as extreme as the value that was actually observed, assuming that the null hypothesis is true (p-value).

If the observations are independent and the population sizes large enough, i.e. $n_{1}+n_{2} \geq 40$ (or instead if the distributions are normal), the two-sample pooled T-test can be used. If the variances in the two populations could be considered as equal, the Student's T-test was used. In the case of unequal variances, the Welch's test was used. Thus, prior to performing one of these two tests, the hypothesis of equality of variances in the two populations was tested using the two-sample Fisher-Snedecor F-test.

When the number of data in the populations was not sufficient to raise the assumption of normality, the non-parametric Wilcoxon's test was used to compare the populations.

Data processing and statistical analyses were performed using the open-source $\mathrm{R}$ software [12].

\section{Results}

\subsection{Validation of the automated cell tracking method}

One of our principal aims is to evaluate the reliability and accuracy of the automated tracking. Although human errors also introduce uncertainties in the results, manual tracking is currently still considered as the 'gold-standard' in cell tracking and used as reference for comparing different cell-tracking systems [14]. We therefore compare it to manual tracking on a sample dataset consisting in 623 trajectories in total.

\subsubsection{Distance between cell positions obtained by manual and automated methods}

The accuracy of the automated method is firstly assessed by computing the distance between the cell positions generated by the automated and the manual tracking of a sub-sample of 282 trajectories.

In Figure 5A, the distance between the position recorded automatically and manually is drawn for every frame. Each line represents the averaged distance between automatic and manual cell positions for each cell of a photographic field (i.e. one line for each of the 36 fields/sequences). The bold lines indicates the mean distance and standard deviation for all cells. As automated tracking involves tracking the movements of cells backwards in time, the error increases slightly from the last to the initial frame. The maximal mean distance is about $26 \mu \mathrm{m}$ which is reasonable given that cell diameter is around $50 \mu \mathrm{m}$ on our photos (cell diameter being roughly considered as the diameter of the round part of the cell). The maximal individual distance reaches about $160 \mu \mathrm{m}$ (Figure 5B), almost certainly corresponding to a situation in which the automatic tracking system lost the target cell. Assuming that a cell is lost by the automated tracking if the distance between automatically and manually generated positions is larger than $50 \mu \mathrm{m}$ in the first frame (which gives the last tracked position for the automated method), a proportion of $4.2 \%$ cells are identified as lost cells. 

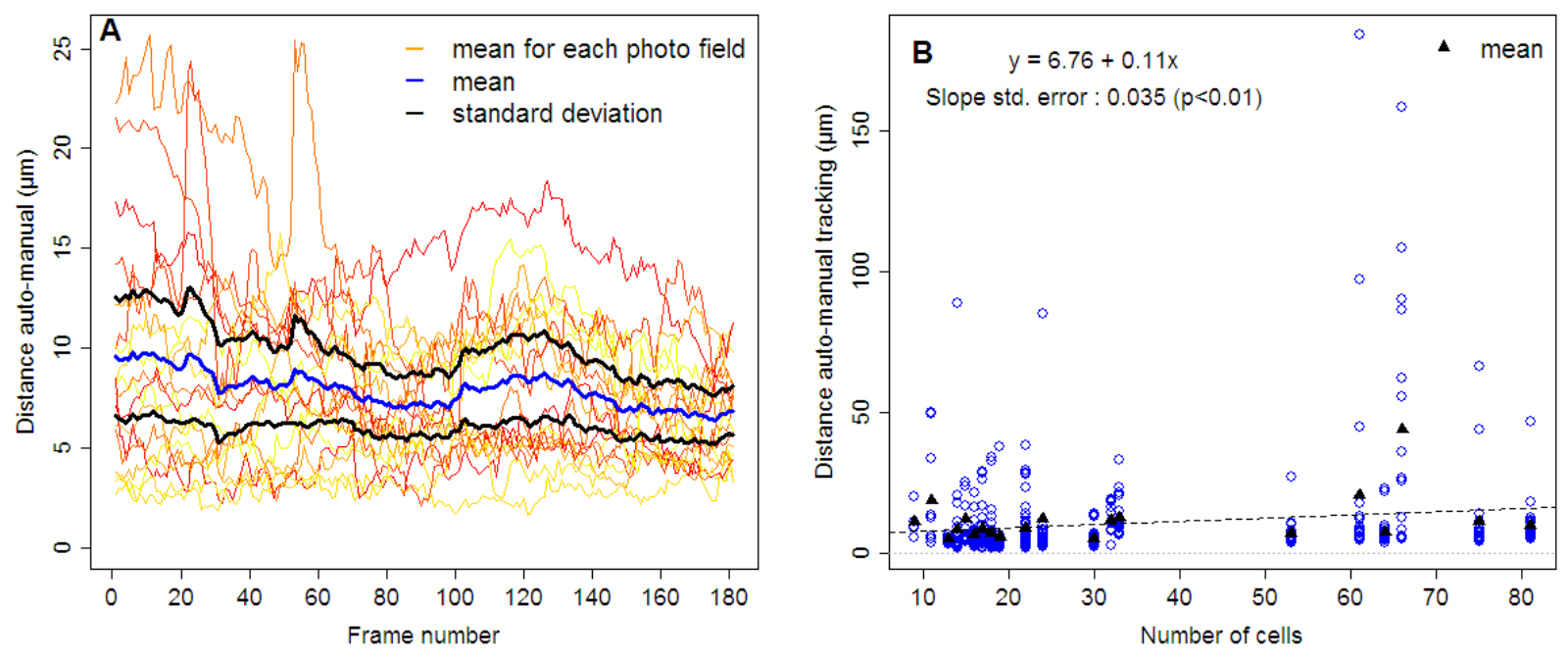

Figure 5: Mean distance between the positions obtained by manual and automatic tracking vs. time (A) and vs. cell density (B).

The variations of the automated-manual tracking distance are also analyzed with respect to the number of cells per frame in order to test whether more cells are lost in higher densities (Figure 5B). For each trajectory, the mean distance between the positions that are provided by the automated and manual methods are represented against the total number of cells in the first frame. A slight but significant increase of the manual-automated distance with increasing cell number is revealed by the linear regression using every trajectory data (slope is significantly positive, $p<0.001$ ) but the mean values at high number of cells is similar to those at low number of cells: mean distance is $11.2 \mu \mathrm{m}$ for $\mathrm{N}=75$ cells and $9.8 \mu \mathrm{m}$ for $\mathrm{N}=81$ cells (black triangles) while it is $11 \mu \mathrm{m}$ for $\mathrm{N}=9$ cells and $18.6 \mu \mathrm{m}$ for $\mathrm{N}=11$ cells. Therefore, it can be concluded that although there are more lost cells in higher densities, there is a good agreement between the positions generated by the automated and manual methods (globally, only $4.2 \%$ of lost cells).

\subsubsection{Consistency of results obtained by manual and automated tracking for the trajectory parameters}

In a second step, the reliability of the tracker is evaluated for the features characterizing the trajectories, as defined above. A sample of 623 trajectories, randomly chosen, is used out of the 3,276 cell trajectories obtained. The number of cells counted in the final frame of each field (at 12 hours), the mean cell speed in each photographic field, the mean MaxDist and the mean FRDO are compared between automated and manual tracking. The results obtained with the two methods are globally consistent as shown in Figure 6.

The position of cells in the last frame of each sequence was marked manually to initialize automated tracking process. Panel A in figure 6 therefore illustrates inter-operator variability for the choice of cells to be considered. The choice may be ambiguous for partially overlapping cells and cells located at the edge of the frame. The values of MaxDist and FRDO obtained were 
A - Number of cells on last frame

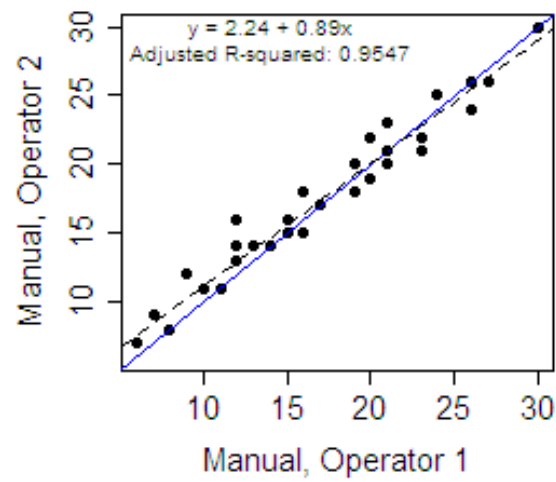

C - MaxDist $(\mu \mathrm{m})$

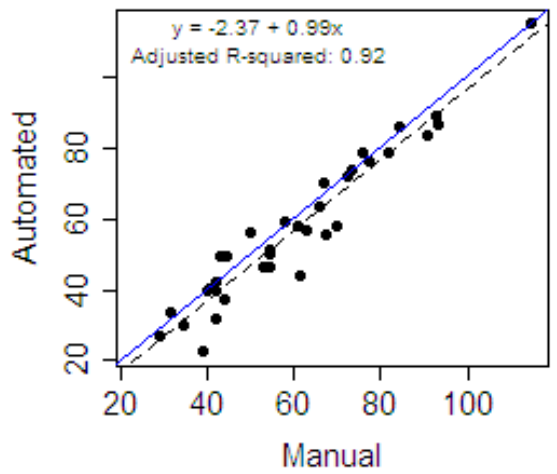

B - Mean speed ( $\mu \mathrm{m} . \mathrm{min}-1)$

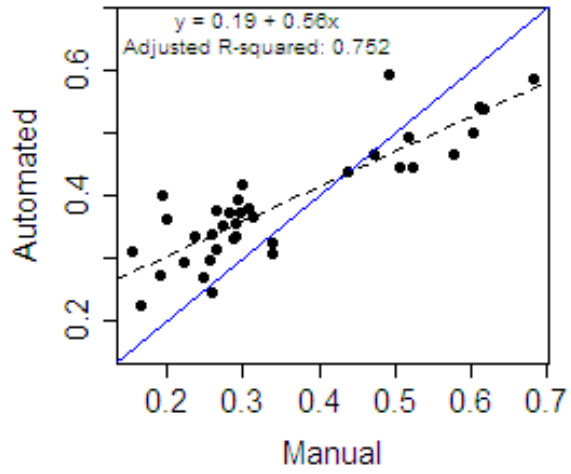

$\mathrm{D}-\mathrm{FRDO}(\mu \mathrm{m})$

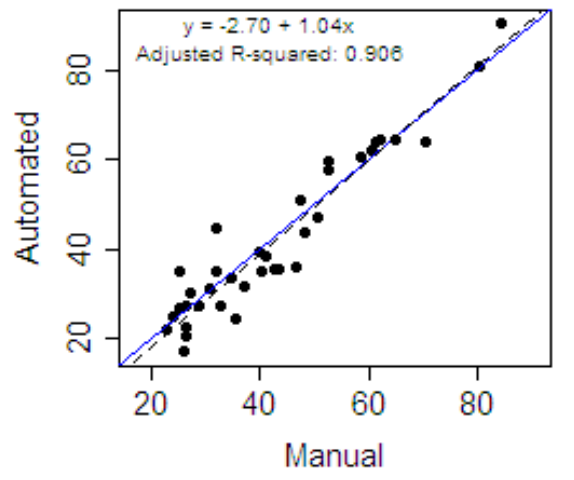

Figure 6: Comparison between the number of cells counted on the final frame by two operators (A), and between automated and manual tracking for mean cell speed per photographic field (B), MaxDist (C) and FRDO (D). Each dot represents the mean of all data of every cell of a given photographic field $(\mathrm{n}=36)$. The blue line is $y=x$ and the dotted line represents the linear regression between the data obtained with the automated and manual methods.

consistent for manual and automated tracking (Figure 6C and D).

The operator performing the manual tracking focuses on the cell soma, a feature they can easily identify, whereas the automatic tracking method is based on detecting the combined soma and hallo pattern which it can robustly detect. This subtle difference in tracked features results in a limited discrepancy between the human obtained position and the automatic one. We have shown in previous works [7] that this discrepancy is not significant with respect to the measured cell movements. Indeed, at low speeds, the automated procedure gives higher MS values than the manual procedure (panel B). In automated tracking, the neighborhood of the previous position is explored at each time step, so even small displacements of a few pixels are taken into account when updating cell position. By contrast, at higher speeds, manual tracking gives higher estimates than automated tracking. This is illustrated in Figure 7A by an example showing the trajectory of the 
same cell followed by automated and manual tracking. The trajectory obtained by the automated tracker is smoother, when the cell moves faster, than that obtained by manual tracking, probably because human operators tend to use a series of discrete movements, whereas the automated tracker follows the cell continuously. We test this hypothesis by comparing the standard deviation (SD) of instantaneous cell speed for automated and manual tracking (Figure 7B). The mean value of the standard deviation of instantaneous cell speed is higher for the manual tracking, and if all the cells of the sample population are pooled, the mean value is $\sigma_{a}=0.32 \mu \mathrm{m} . \mathrm{min}^{-1}$ for the automated method and $\sigma_{m}=0.42 \mu \mathrm{m} \cdot \mathrm{min}^{-1}$ ( $31 \%$ higher) for the manual method.

\section{A- Manual vs. Automated tracks}

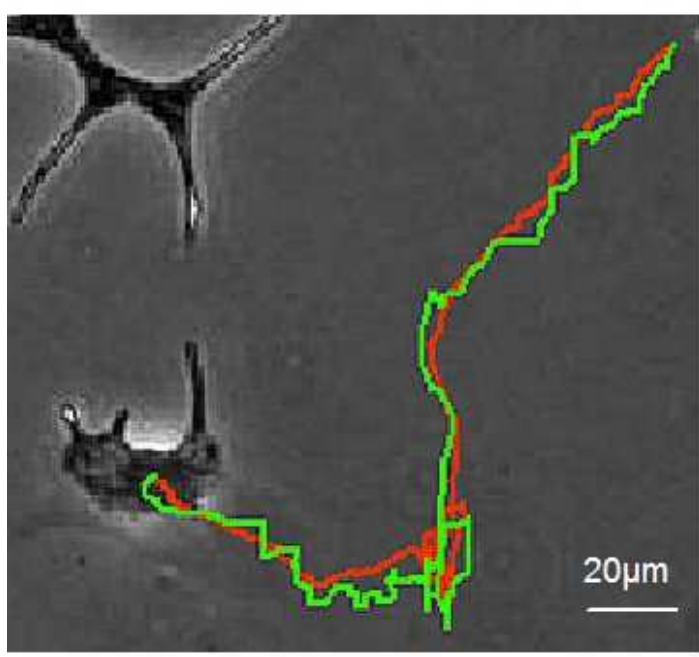

\section{B - Mean value of SD of cell speed ( $\mu$ m.min-1)}

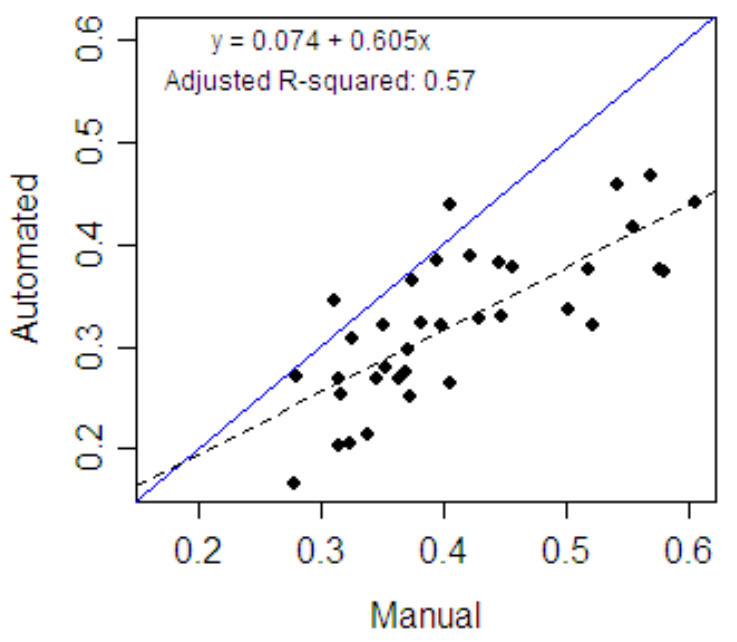

Figure 7: Illustration of the difference in smoothness between automatically and manually tracked trajectories: (A) Trajectories of a same cell detected by automated tracking (red) and manual tracking (green); (B) mean standard deviations of instantaneous cell speed for each photographic field. The trajectory tracked by the automated method is smoother than that tracked by the manual method.

In view of these results, we consider that the agreement between automatically and manually generated data for the indicators of trajectory characteristics is satisfying enough to perform further analyses on the whole dataset.

\subsection{Quantification of cell motile behaviour}

\subsubsection{Comparison of WT and bcat* cells}

The migration characteristics of the two cell lineages are compared on the basis of the features defined above (MS, MaxDist and FRDO), as presented in Table 1.

Data from three experiments, for automatically tracked cells, are pooled for the analysis. To avoid biases due to different recorded durations, only cells that were tracked from the last to the 
first frame (70\% of the total in average) are considered (i.e. cells that moved out or in the frame are not taken into account). Moreover, cells that divided during the experiment are also not taken into account, so that the potential effects of cell division on cell migration may not interfere with the results. This precaution is justified by the results presented in paragraph 4.3.4. that indeed cell speed changes during the division process and in paragraph 4.3.1. that the division rates of bcat* and WT cells differ. After this selection, 57\% of the total number of cells was kept for the analysis. The number of remaining data was still high enough to apply the statistical tests as described in paragraph 3.3.: it varies from 212 to 245 for WT melanocytes and from 377 to 422 for bcat* melanocytes. Fisher-Snedecor F-test shows that the variances differed significantly between the two cell lineages in every group except so the statistical significance is determined using the Welch's test (see paragraph 3.3.).

Table 1: Comparison of motility and migration parameters between WT and bcat* cell lines, for each fibronectin concentration. The statistical significance levels were performed using Welch's test to quantify the difference between WT and bcat* cell lines. Cells that divided or that moved out the field were excluded from the analysis. Mean values for each population are given along with the standard deviations between brackets. The bold font indicates the group with the highest mean value.

\begin{tabular}{|c|c|c|c|c|c|c|}
\hline \multirow[t]{2}{*}{ Coat } & \multicolumn{2}{|c|}{$\mathrm{MS}\left(\mu \mathrm{m} \cdot \min ^{-1}\right)$} & \multicolumn{2}{|c|}{ MaxDist $(\mu \mathrm{m})$} & \multicolumn{2}{|c|}{ FRDO $(\mu \mathrm{m})$} \\
\hline & WT & bcat* & WT & bcat* & WT & bcat* \\
\hline none & $0.282(0.088)$ & $\mathbf{0 . 2 8 6}(0.112)$ & $45.2(27.4)$ & $\mathbf{4 7 . 6}(30.8)$ & $29.4(23.6)$ & $35(29.2) * *$ \\
\hline $1 \mu \mathrm{g} / \mathrm{ml}$ & $0.298(0.09)$ & $\mathbf{0 . 3 0}(0.104)$ & $47.8(28.2)$ & $51.2(34.8)$ & $28.6(24.2)$ & $38.4(33) * * *$ \\
\hline $40 \mu \mathrm{g} / \mathrm{ml}$ & $0.408(0.158)$ & $0.372(0.172) * *$ & $91(60.4)$ & $67.2(39.2) * * *$ & $64.8(59)$ & $50.4(37) * * *$ \\
\hline
\end{tabular}

The statistical analysis presented in table 1 shows that, for cells grown on uncoated conditions, the differences between the two lineages are found to be significant for FRDO $(p<0.01)$, the FRDO values being around 20\% higher for bcat* cells than for WT cells. The mean speed and MaxDist are similar for the two cell lineages. It thus shows that bcat* cells are prone to migrate over greater distances than WT cells. In other words, WT cells tend to stay in their initial neighbourhood, whereas bcat* cells show a trend of migrating and staying away from their initial position, implying a difference in underlying mechanisms affecting their migratory behaviour (since they have similar MS but higher FRDO).

Similar results are observed for low coating conditions (FN 1): compared to WT cells, bcat* cells have higher FRDO values but similar MS and MaxDist which means that bcat* cells show similar motility (characterized by MS or by the total length of the cell trajectory, which is equivalent) but higher migratory activity (characterized by the final linear displacement of the cell). These two features are further detailed in paragraph 4.4. and illustrated in Figure 14.

In contrast, for the $40 \mu \mathrm{g} / \mathrm{ml}$ fibronectin concentration, the trend is reversed and all features are significantly different: bcat* cells express lower motility and migratory activity than WT cells ( $p<0.01$ for MS; $p<0.001$ for MaxDist and FRDO). These results highlight the impact of the matrix substratum on cell movements: it shows that the effect of fibronectin (at the higher 
concentration) is more important for WT cells than for bcat* cells.

\subsubsection{Influence of fibronectin coating}

The same three parameters - MS, MaxDist and FRDO - are also quantitatively analyzed in relation with the fibronectin concentration, using the same dataset. Fisher-Snedecor F-test on variances equality between the two populations authorizes the use of the Student's T-test only for the comparisons between FN0 and FN1 coating conditions; otherwise the Welch's test is used. In each cell line, the results in coated conditions are compared to the uncoated condition that is considered as the reference (Figure 8). For the two cell lines, no significant change is observed between uncoated and low fibronectin $(1 \mu \mathrm{g} / \mathrm{ml})$ conditions, whereas significant increases are found for the highest concentration, $40 \mu \mathrm{g} / \mathrm{ml}(p<0.001)$.
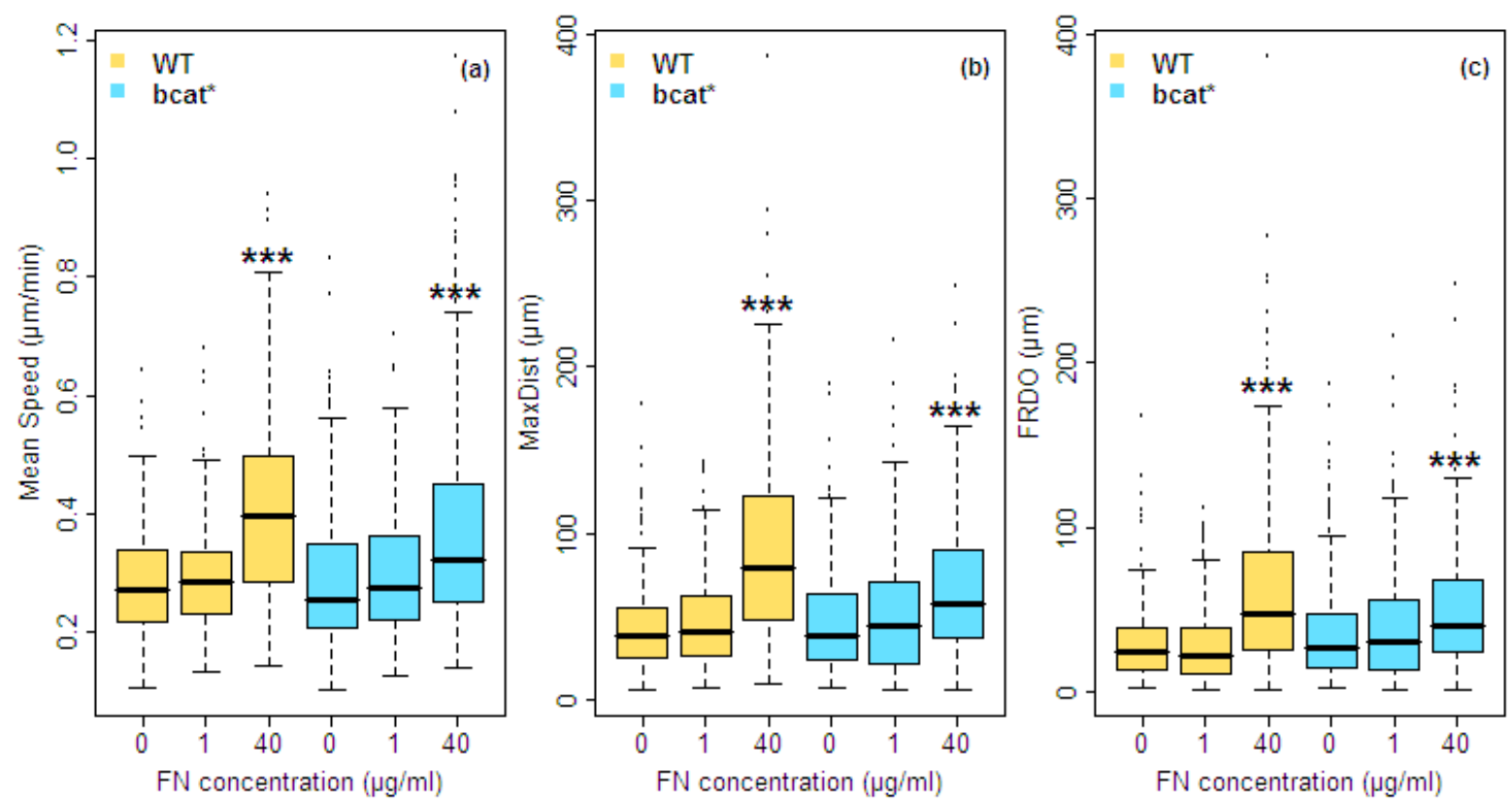

Figure 8: Parameters of cell motility in each cell lines, WT and bcat*, in relation with the concentration of fibronectin: (a) mean speed, (b) trajectory amplitude and (c) Final relative distance to origin. The effects of the fibronectin concentrations are quantified by comparison to the control condition (uncoated) for each cell line using Welch's and Student's tests (***: $p<0.001)$.

\subsection{Cell division and its influence on migration}

\subsubsection{Influence of $\beta$-catenin on cell division}

The division rate is compared between WT and bcat* cells grown on uncoated plate-dish. The division rate is calculated by dividing the number of cell divisions during the experiment time span by the initial number of cells in the field and is expressed as a percentage of the initial cells. The 
variances of the two groups are not significantly different (F-test p-value is 0.56 ) and the numbers of cells are $n_{W T}=355$ and $n_{b c a t *}=814$. A two-sided Student's T-test is applied and confirms the increase of division rate for bcat* cells observed in Figure 9. The percentage of cells that divide during the experiment time is $17.8 \%$ for bcat* cells while it is $6.8 \%$ for WT cells.

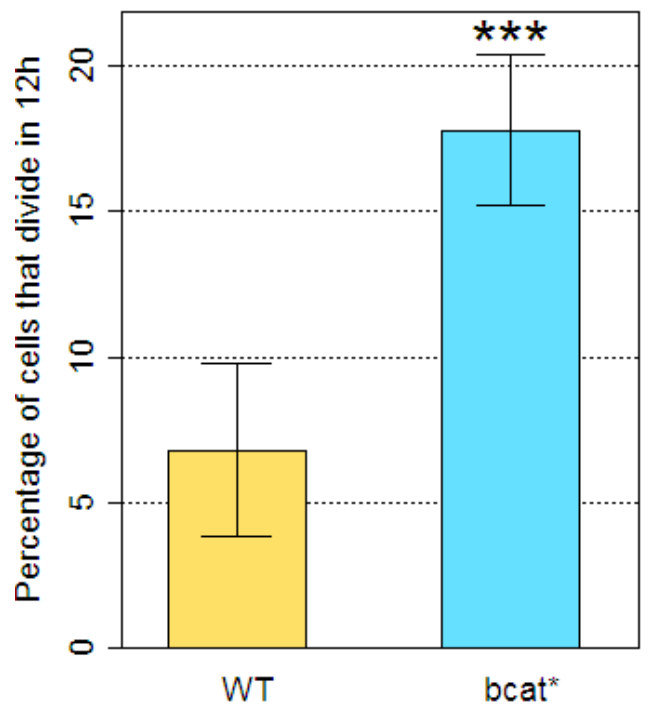

Figure 9: Division rate for each cell line in uncoated condition. The statistical significance levels were calculated using a two-sided Student's T-test $(* * *: p<0.001)$.

\subsubsection{Influence of density on cell division}

To investigate the effect of cell crowding, we analyze the rate of cell division during the $12 \mathrm{hr}$ experiment as a function of the density of cells in the frame (Figure 10). We choose to use bcat* cells only for this analysis, as the number of divisions for WT cells is too low to provide reliable estimates of the division rate.

Cell density within the field has very little effect on division rate. Linear regressions are carried out for each coating condition, not in order to assess the existence of a linear correlation between the variables but simply to extract general trends of variations. The obtained slopes are close to zero: only one value is significantly different from zero $(p<0.05$, T-test $\mathrm{p}$-value for no coating condition). More particularly, the estimated slopes are all positive, which means that no decrease in the division rate is observed with increasing cell density, as would have been expected if competition for space was a major factor (within the range of density values of our set of data).

\subsubsection{Influence of fibronectin coating on cell division}

The division rate (percentage of cells that divide during the experiment time span) is compared for the three different culture conditions: uncoated, coated with $1 \mu \mathrm{g} / \mathrm{ml}$ and with $40 \mu \mathrm{g} / \mathrm{ml}$ (Figure 11). 


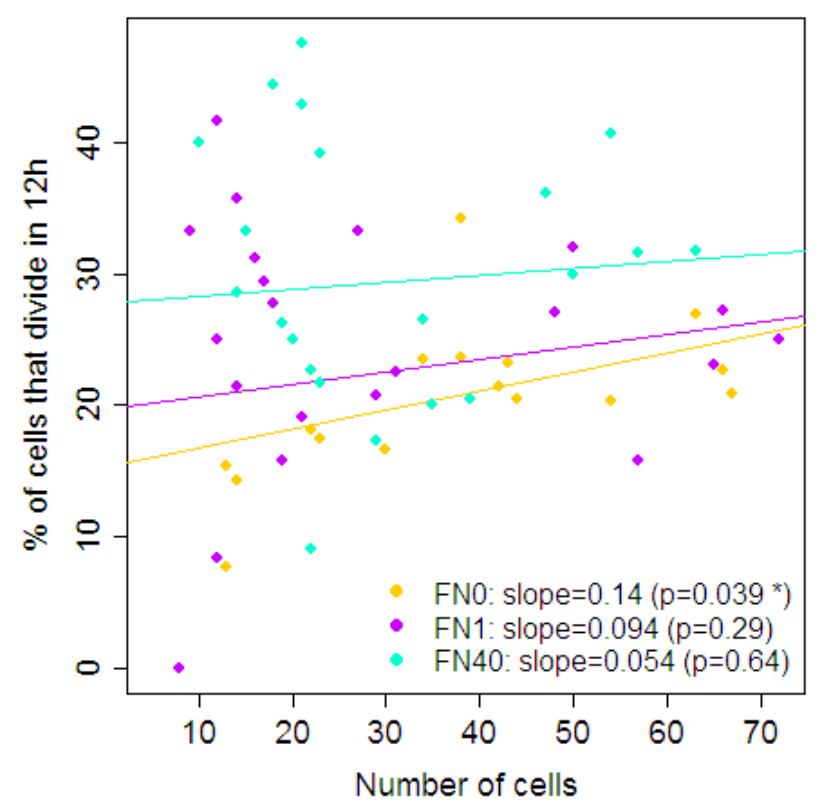

Figure 10: Percentage of cells dividing during the experiment (12h) as a function of the total number of cells in the photographic fields. These data were obtained from three independent experiments. We use the bcat* cell lineage for this study, as this lineage displays the widest range of cell densities in our experiments. Lines represent the linear regressions for every coating condition: no coating (FNO), coating with low fibronectin concentration (FN1) and coating with a higher fibronectin concentration (FN40). Slopes of the linear regressions are displayed along with a twosided T-test $\mathrm{p}$-value for testing a significant difference to zero.

The variances of each group are not found significantly different according to the results of F-tests (p-values higher than 0.3). The numbers of individuals range from 355 to 399 for WT cells and from 714 to 814 for bcat* cells. Therefore a Student's T-test is performed to assess the significance of the effect of coating compared to the uncoated control for each cell lineage. No significant change in the division rate is found when switching from uncoated to coated with $1 \mu \mathrm{g} / \mathrm{ml}$ fibronectin conditions (p-values of 0.83 and 0.72 for WT and bcat* cells respectively). A slight effect is found for the highest concentration of fibronectin: $p<0.05$ and $p<0.1$ for WT and bcat* cells respectively. Figure 11 shows that division rate variability is in fact more linked to cell lines than to the coating conditions: it is an intrinsic property of cells. Indeed, this is illustrated in Figure 9 for the uncoated condition, and regarding the coated conditions, the Welch's tests give the same result $(p<0.001$ for the two fibronectin concentrations and for the uncoated condition, not shown). It can be concluded that division rates are significantly higher for bcat* cells than for WT cells regardless of the coating conditions. 


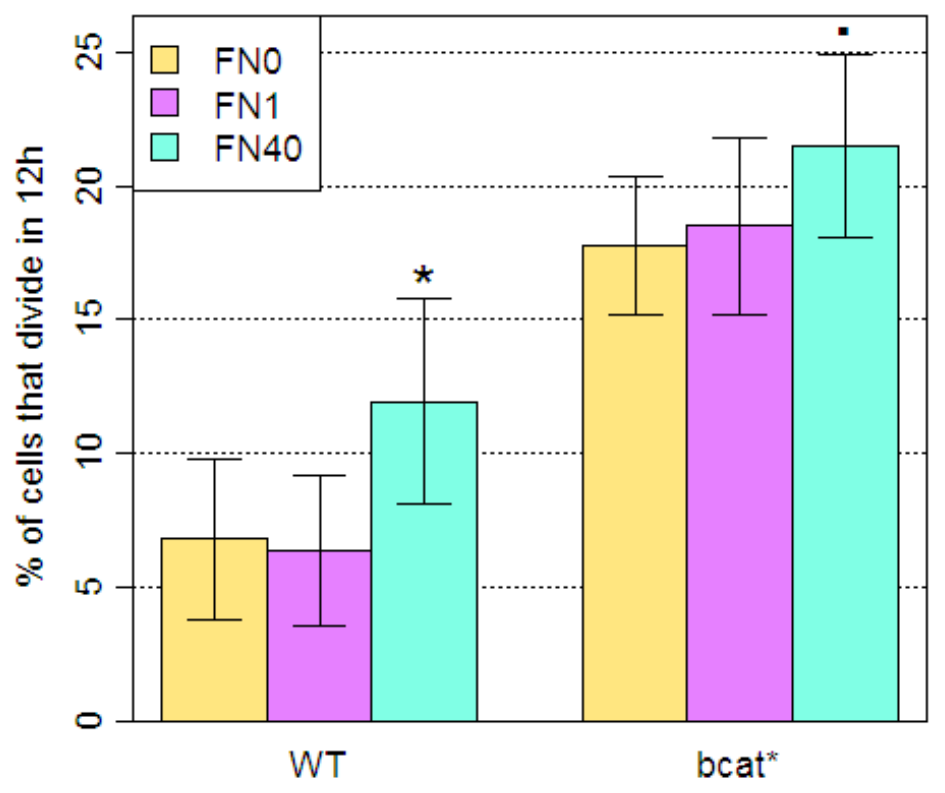

Figure 11: Mean percentage of cells that divide during the experiment time span (12h) on every photo field. Data are pooled from three experiments. Each group of bars represents one cell lineage. Colours correspond to the different concentrations in fibronectin (FN0, FN1, FN40). The significance levels are computed using a two-sided Student's T-test $(: p<0.1 ; *: p<0.05)$

\subsubsection{Influence of division on cell speed}

The data collected is then used to study the variation in cell speed when there was a division. The profile of cell speed generally reaches a maximum just after a division event, as illustrated in Figure 12.

For $60 \%$ of the cells that divided during the experiment, the maximal recorded speed value is observed during the ten frames following the time of division. Figure 12 represents the sequences of instantaneous speed $\left(S_{c}(t)\right)$ over time for four mother cells and the four couples of daughter cells issued from divisions. These sequences are arbitrarily chosen for a purpose of illustration: three of them are taken from the pool of bcat* cells grown on plastic (uncoated) and the fourth one is selected from the WT cells grown on coated conditions (FN1). The sequences are aligned on the division time $(\mathrm{t}=0)$, which means that if a cell divides at half experiment, its speed values are reported in the interval $\left[-t_{f} / 2, t_{f} / 2\right], t_{f}$ being the experiment time span (720 minutes). Note that the scale of the Y-axis is different for each sub-graph, as these sampled cells have different range of speed values, but the position of the maximum is yet a stable pattern.

To quantify this effect, means of instantaneous speeds are computed on the population of cells that divided during the experiment, the sequences being aligned on the division time $(\mathrm{t}=0)$, as repre- 


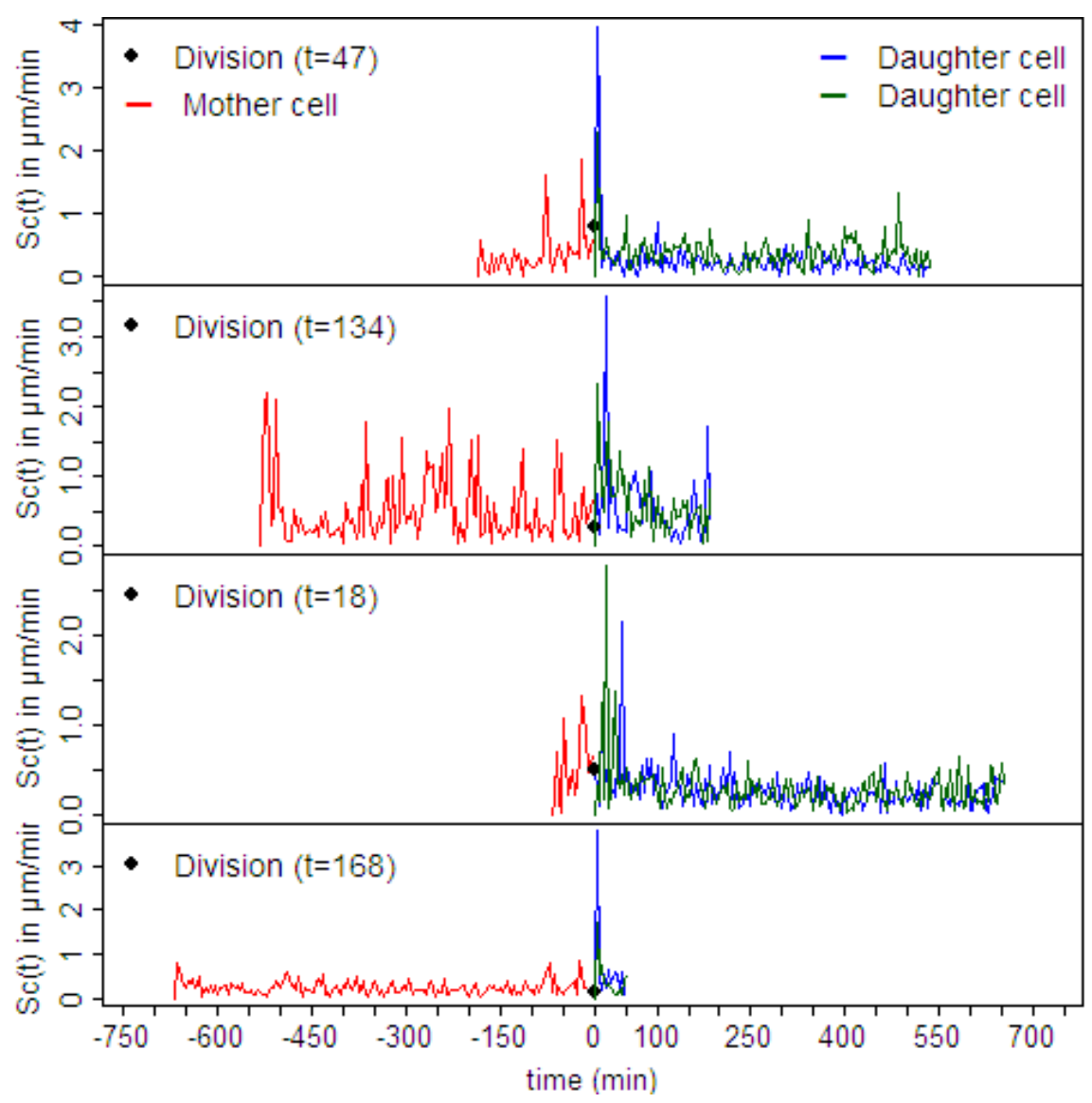

Figure 12: Instantaneous speed $S_{c}(t)$ over time for four mother cells (in red) and the four associated daughter cells (in blue and green). The sequences are aligned so that the division occurs at $\mathrm{t}=0$. The instantaneous speed values are generally maximal just after division.

sented in Figure 12. Figure 13 shows the means and standard errors, in red for mother cells (before division) and blue for daughter cells. The black line represents the number of cells considered in the calculation at each time step. The number of cells to consider decreases from $\mathrm{t}=0$ to $\mathrm{t}=t_{f}$ and from $t=0$ to $t=-t_{f}$ since each speed sequence only covers half of the whole time interval (see Figure 12 for an illustration). The total number of mother cells is 378 and the number of daughter cells is 613 (the number of cells is not exactly double the number of mother cells, because divisions are taken into account only if the mother cell was tracked while some daughter cells might not be tracked, for instance if they moved out of the frame). Instantaneous cell speed increases rapidly during the division process, for presumably different reasons in the cases of mother and daughter cells. For mother cells, the increase of speed can be provoked by the change of cell shape and size: cells shrink before division and therefore their gravity center, and the tracked position, can be displaced. Just after division, daughter cells quickly move away which also induces the observed increase of speed values. After division, the daughter cell speed decrease slowly: two hours after the division, the mean speed of daughter cells is still significantly higher than that of mother cells 

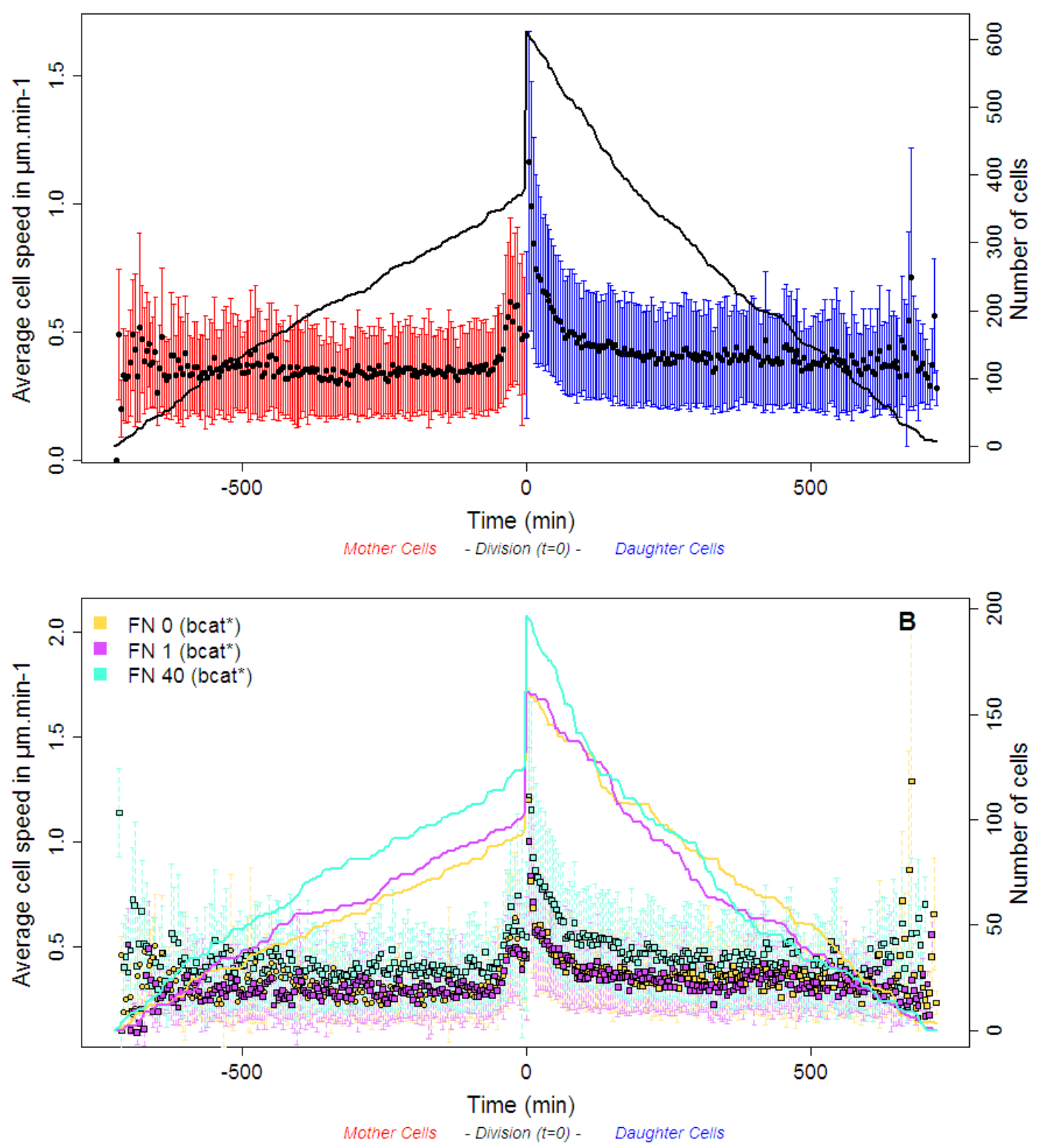

Figure 13: (A): Variations of cell speed in relation with the phases of the division process: mean and standard deviation of cell instantaneous speed with respect to time, the origin being placed at the division time. The black line represents the number of cells that was considered to compute the mean value at each time step. Just before division (in red), cells are in the phase G2 of the cell cycle while the cells in blue are in the phase G1. (B): Same graph but distinguishing the different coating conditions, for bcat* cells only.

(Welch's test, $p<0.001$ ). Means of cell speeds are $0.352 \mu \mathrm{m} \cdot \mathrm{min}^{-1}$ and $0.402 \mu \mathrm{m} \cdot \mathrm{min}^{-1}$ on the time intervals [-520min; -120min] and [+120min; +520min], i.e. for mother and daughter cells 
respectively.

To show how the coating conditions modify that speed burst after division, the same dataset is represented in Figure 13B but for each fibronectin concentration. Only bcat* cells are considered since the division rate of WT cells is not high enough to provide reliable results, due to the large variability of the data. In uncoated conditions or coated with low fibronectin concentration, the speed profiles are similar while at $40 \mu \mathrm{g} / \mathrm{ml}$ fibronectin coating, cell speed remains high longer after the division. For the three conditions, the changes in cell speed appear approximatively 40 minutes before the division time. Therefore, the average cell speed in the stable phase before division is calculated using data on the interval [-640min; -40min] (the first values are not considered since the number of cells is very low): $0.30 \mu \mathrm{m} \cdot \mathrm{min}^{-1}$ for FN0 and FN1, and $0.40 \mu \mathrm{m} \cdot \mathrm{min}^{-1}$ for FN 40. In average, the increase of cell speed during the two hours after division is of the same range for the three conditions: average values on the interval $[0 ; 120 \mathrm{~min}]$ are $0.50,0.49$, and $0.68 \mu \mathrm{m} \cdot \mathrm{min}^{-1}$ for FN0, FN1, and FN40, which correspond to relative increases of respectively $64.0 \%, 67.1 \%$ and $68.7 \%$ compared to the reference cell speeds before division.

Several biological mechanisms are involved in this interaction between cell division and migration. Cells must be polarized to migrate and their migration follow their polarity $[27,5,19]$. Cells have also polarity during mitosis due to the orientation of the microtubule-organizing centers where microtubules elongate, and the two poles move away from each other to two opposite sides [1]. Cell membrane pinches in to form cleavage furrow, resulting in two daughter cells which quickly move to the opposite side. The challenge is the evaluation of forces involved in cellular processes and understanding their roles. Studies measuring or modifying forces suggest biochemical and mechanical signals, and links with the substrate [31, 32]. Mechanotransduction signals might be involved in the control of the very last step of cell division.

\subsection{Influence of cell shape}

Melanocytes are usually round or oval, but fusiform and dendrite shapes are also recognized, as expected from cells derived from the neural crest. Melanocytes cultured with keratinocytes induce a highly dendritic phenotype with extensive contact between the two types of cells, which allow a melanosome transfer from melanocytes to keratinocytes [18, 29]. In melanocyte cultures in vitro, contact is observed between melanocytes, but the role of these contacts is unknown.

In our experiments on wild-type melanocytes in uncoated wells, we occasionally observed extensive contact between dendritic melanocytes which formed multicellular aggregates or clusters (Figure 14A). These dendritic melanocytes are motile but non-migrating, i.e. they do not show any displacement outside of the cluster and have a low MaxDist and FRDO. However, this seems not due to a high cellular density. Interestingly, in vivo during the first hours following their separation from the neural tube, crest cells remain poorly spread and form some clusters [10]. However, these observations were done in chicken and must still be confirmed in mammals. In contrast, on fibronectin, cell shapes are altered and cells usually spread and migrate with preferential direction (Figure 14B).

Two types of trajectories as a function of cell shape are observed in the melanocytes of bcat* 

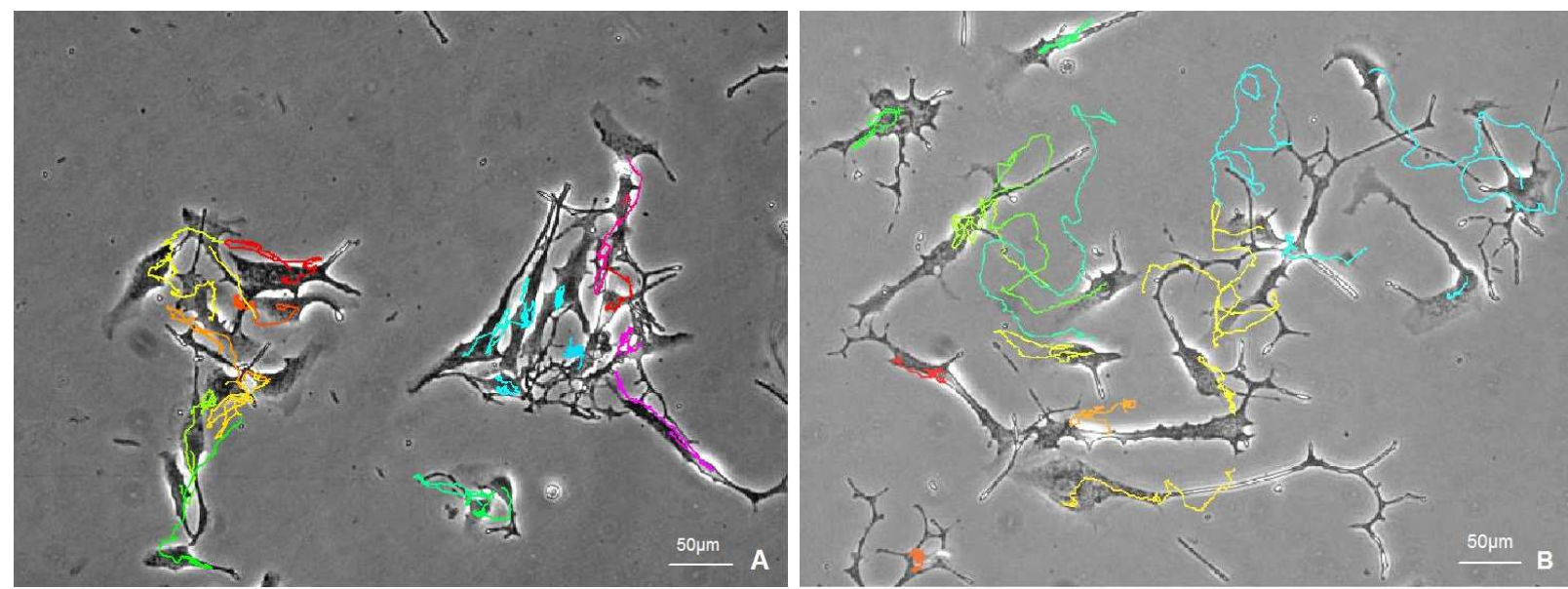

Figure 14: Trajectories of wild-type dendrite melanocytes cultured on plastic dish without any coating forming aggregates, motile but non-migrating (A) or cultured on plastic dish with a coating of $40 \mu \mathrm{g} / \mathrm{ml}$ fibronectin (B). In this condition, aggregates disintegrate, the cell shape of melanocytes change and melanocytes migrate with preferential direction.

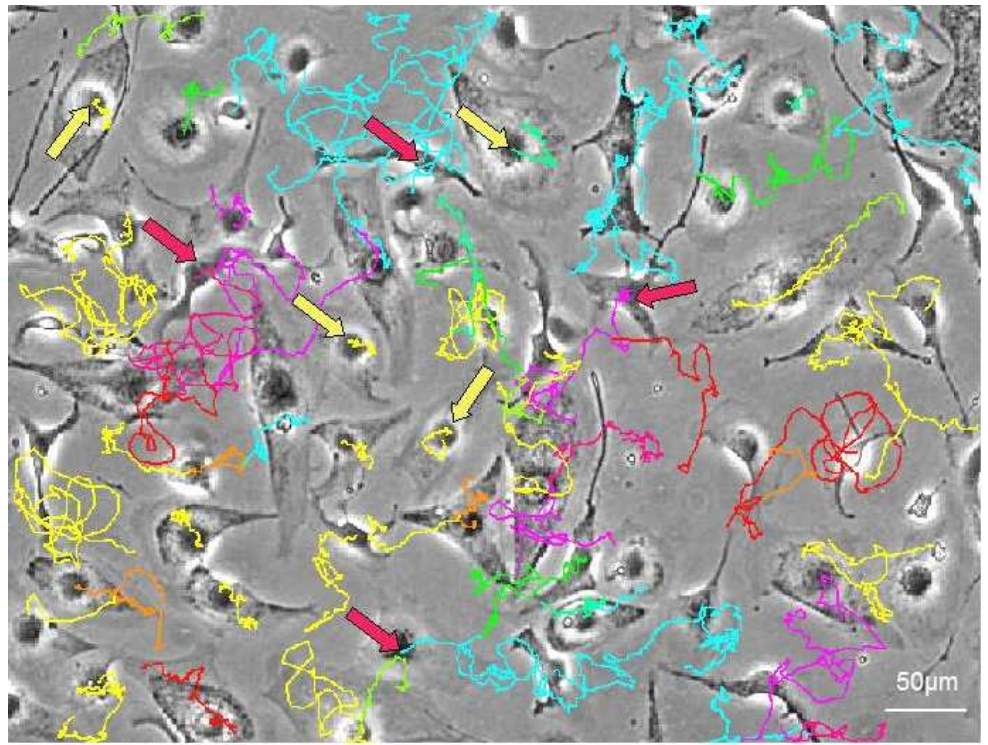

Figure 15: Cell shape and cell trajectories for bcat* melanocyte cell line cultured with coating $40 \mu \mathrm{g} / \mathrm{ml}$ illustrated by time-lapse image sequence. Large expanded cells associated to very small displacement are indicated with yellow arrows. Thin cells with dendrites associated with long displacements in preferential directions are indicated with red arrows.

cell line cultured on $40 \mu \mathrm{g} / \mathrm{ml}$ fibronectin. The first type is characterized by a high trajectory amplitude and a total displacement linked to a cell shape comparable to that of wild-type melanocyte and with migration patterns with preferential direction. The second type appears as large spreaded cells associated with no or very small displacement (Figure 15). 
This last type is only observed in melanocytes over expressing $\beta$-catenin and might contribute to the decrease of the mean speed of these cells (Figure 16).
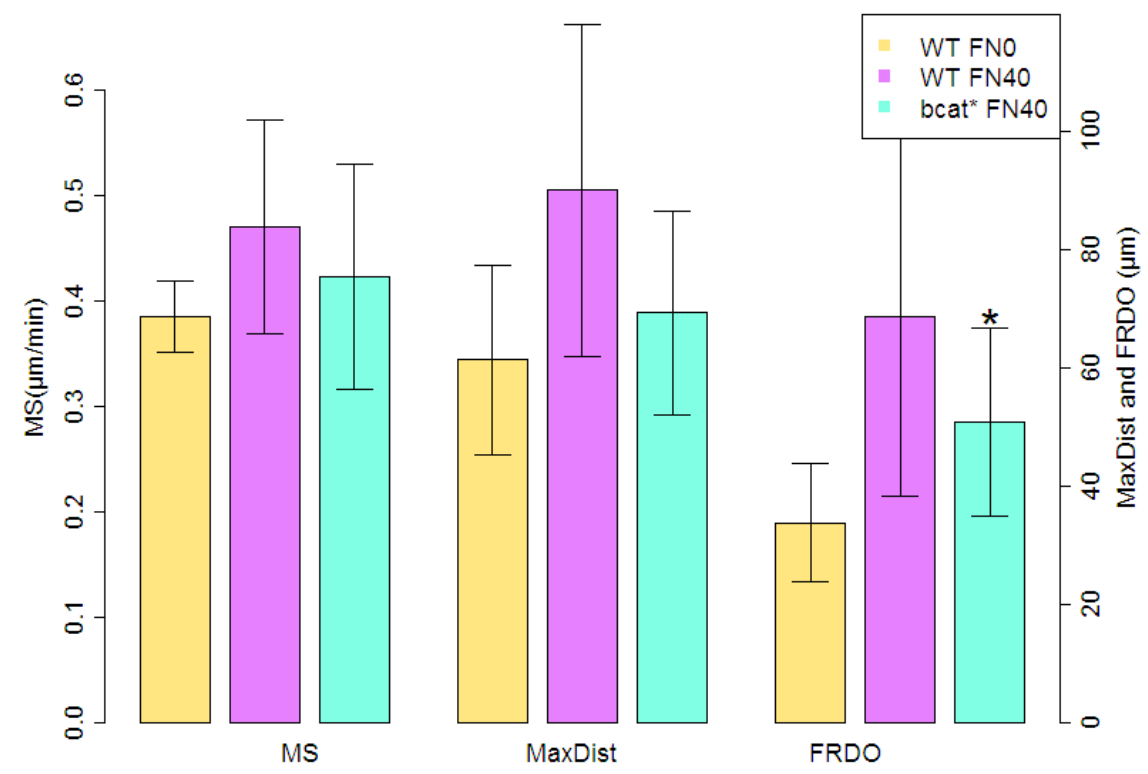

Figure 16: Comparison of mean speed, trajectory amplitude, and final relative distance to origin for the three particular sequences presented in the photos: dendrite melanocytes cultured without fibronectin coating (FN0, Figure 141) or with coating (FN40, Figure 14B) and dendrite melanocytes cultured with high concentration of fibronectin coating (FN40, Figure 15). Significance statistical levels were performed using the non-parametric Wilcoxon's test for WT FN40 (number of data is around 15 which is not enough to raise the assumption of normality) and the Welch's test for bcat* FN40 (n=49).

The comparison of motility performances, such as MS, MaxDist and FRDO, in WT melanocytes cultured without any coating (FNO) and WT melanocytes cultured with a coating of $40 \mu \mathrm{g} / \mathrm{ml} \mathrm{fi-}$ bronectin confirm the biological observations described beforehand. One possibility is that fibronectin partially alters cell shape based on its ability to mediate attachment.

\subsection{Influence of the cell density}

The trajectory characteristics are analyzed with respect to the cell density, defined as the initial number of cells per frame. Figure 17 shows the MS, mean MaxDist and mean FRDO for each photographic field. All datasets are used, regardless of the conditions or cell lines. For the three variables, the dispersion is higher in low densities than in high densities. The graphs reveal no particular trend: the slopes of the linear regressions are 0.0002 (standard error 0.0006), 0.013 (standard error 0.13) and 0.080 (standard error 0.11) for MS, MaxDist and FRDO respectively. None of them is found significantly different from zero and none of them is negative. These 
results suggest that the cell density (number of cells per frame) is not high enough to prevent cell movement.
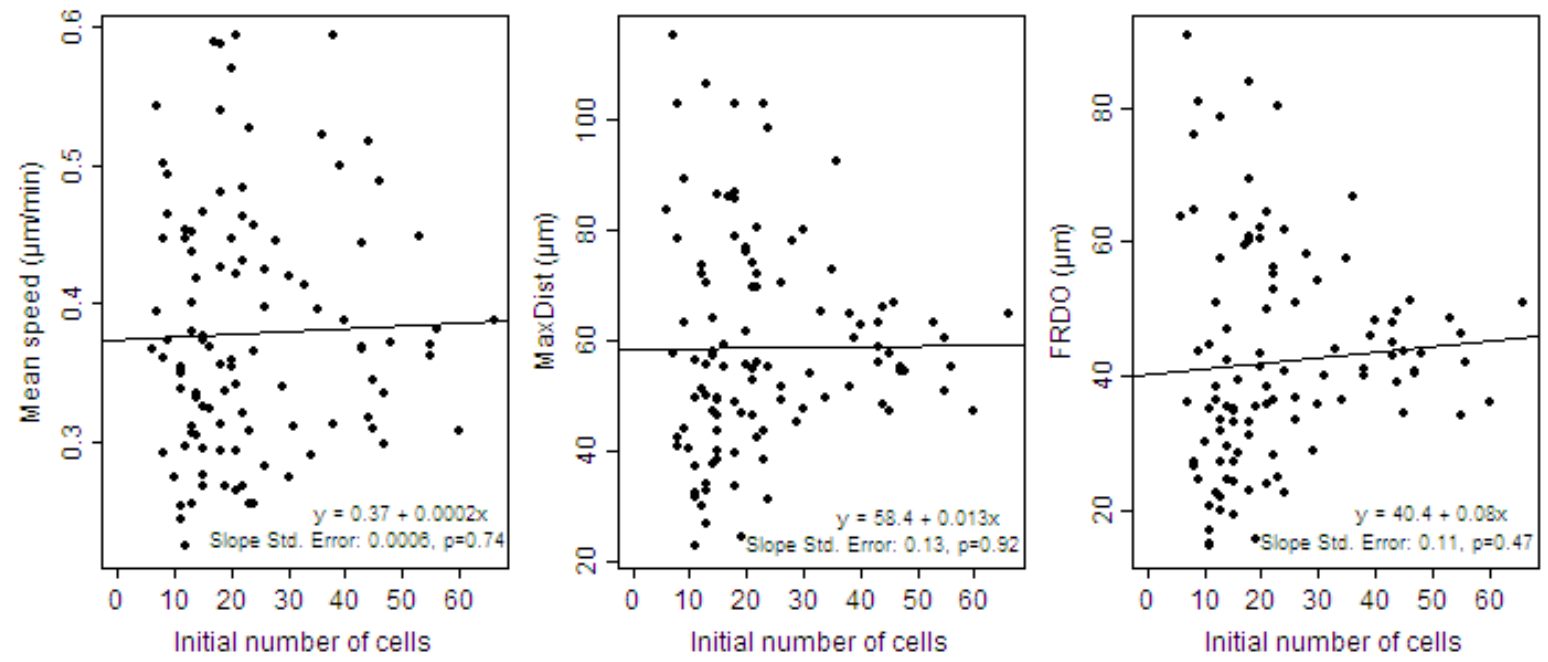

Figure 17: Mean speed (MS), trajectory amplitude (MaxDist) and final relative distance to origin (FRDO) with respect to the initial number of cells per frame for every experiment. Lines represent the linear regression used to analyze the potential existent trend.

To highlight the combined influence of cell density, cell line and fibronectin coating, linear regressions are then performed on each combination (cell line x coating condition), Figure 18.
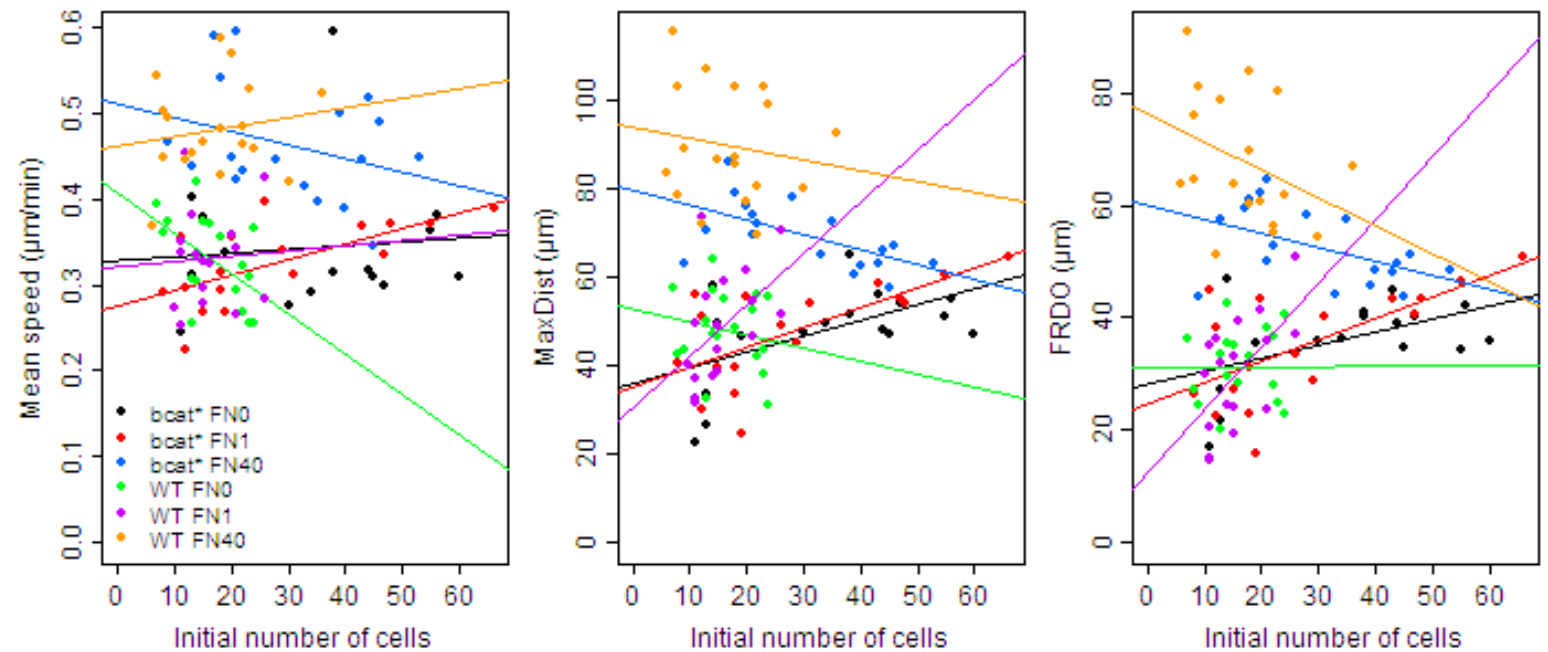

Figure 18: Mean Speed (MS), trajectory amplitude (MaxDist) and final relative distance to origin (FRDO) with respect to the initial number of cells per frame for every experiment with linear regression for each combination cell lines $\times$ coating conditions. 
Table 2: Results of the linear regression for each combination of cell lines and coating conditions: slopes, standard error and significance level of the difference to zero.

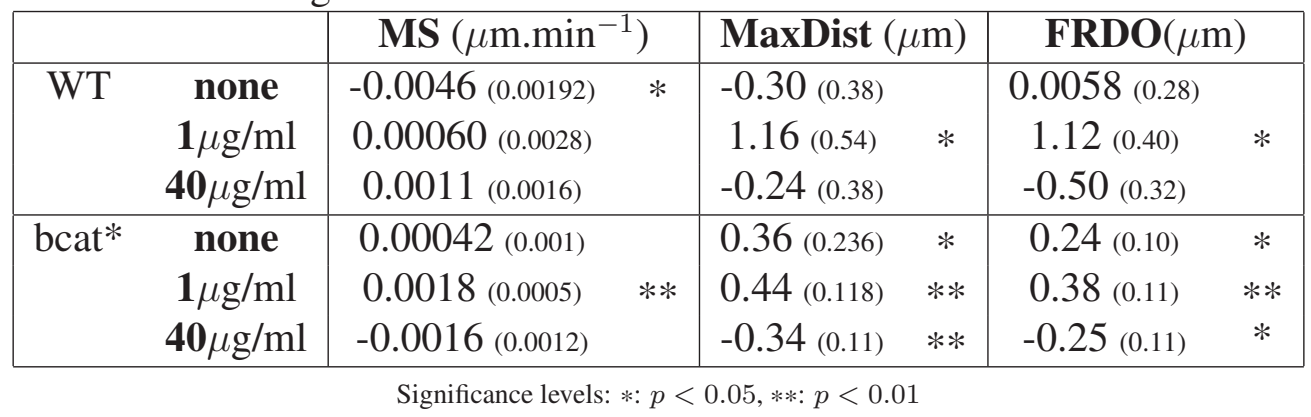

Table 2 presents the slope coefficient and the corresponding standard error (between brackets) of each linear regression, along with the significance of the difference of the slope value to zero. The number of data is $n=18$ for each regression. The regression slopes are not significantly or just slightly different from zero for WT cells (for each variable, only one slope was significantly positive, which might not be very representative of a real trend). However, no definitive conclusion can be drawn from these data since the number of cells per field rarely exceed 30 for WT cells. Regarding bcat* cells, the slopes of the three migration criteria (MS, MaxDist and FRDO) are positive for FN0 and FN1 and negative for FN40. This can be related to the higher division rate of bcat* cells cultured on a fibronectin coating of $40 \mu \mathrm{g} / \mathrm{ml}$ : the number of cells reaches higher values and therefore the effects of crowdedness might begin to be visible. It is interesting to see that this stifle effect is less pronounced for MS than for the two other variables. In high densities, cells conserve their MS values while their values of MaxDist and FRDO are diminished.

To summarize, the main results that can be derived from the data analysis are presented hereafter. First, bcat* cells have a higher division rate (Figures 9 and 11). Second, cell speed increases during the division process and daughter cell speed stabilizes slowly (Figures 12 and 13). Third, the rate of cell division is not diminished in higher cell densities (within the range of density values of our data set). Cell density also has a weak influence on cell migration and motility for bcat* cells at low fibronectin coating conditions only. In contrast, at high fibronectin concentrations and at increasing cell density, bcat* cells maintain similar mean speed values while their final relative distances to origin are diminished (Figure 18). Lastly, for the two cell lines, fibronectin coating significantly increases cell motility and migration only at the higher concentration (Figure 8). Compared to WT cells, bcat* cells have higher motility and migratory ability when cultured in uncoated or low coating conditions. In contrast, for the high fibronectin concentration, the trend is reversed: bcat* cells express lower motility and migratory abilities than WT cells (Table 1 and Figure 8). 


\section{Discussion and conclusion}

Understanding cell migration and its related biological processes requires a dynamic method of data acquisition. Time-lapse video of phase-contrast microscopy provides an interesting noninvasive method of measurement, for experiments studying cell migration in vitro. Unfortunately the high variability inherent in any living biological system and especially in in vitro cell migration systems requires the collection of a large amount of data to reveal changes in cell behaviour [15]. The automated cell tracking method based on the mean-shift algorithm [7] can help fulfill this requirement.

The first step in our analysis of in vitro melanocyte migration was to assess reliability of this method on our experimental data by comparison with manual tracking on sample data. Some visual problems have been detected when considering the results of the automated tracking: the track of a cell may be lost due to cell overlapping, close proximity to neighbouring cells or to debris in the background that may be mistaken for cells. When a cell is lost, the cell tracker usually 'floats' around and records a non relevant trajectory. These problems might be due to the low contrast of the the images and to the specific shape of cells from the lineage in our study: most cells (about $60 \%$ ) are very long and thin with several dendrites, as illustrated in Figure 14. For this class of cell, it happens that the position of the tracking model moves along the cell dendrites when the cell shape varies. Indeed the nuclei are hardly visible and the cell tracking model of cell detection based on a combination of triangles organized in concentric pattern has difficulties to adapt to the cell shape.

Another difficulty is that, because cells are tracked from the last frame to the first one (backward time direction), cells that are leaving the field of view (in forward time direction) do not exist on the last frame and therefore are not tracked. On the other hand, cells that enter the field of view during the sequence and that are present until the last frame, are tracked $(23 \%$ in average enter the field in our experiment). The amount of trajectories entering or leaving the field of view is limited with respect to the total amount of trajectories and furthermore, in order to make motility measures independent of the duration of tracking, the motility features (MS) are normalized by the number of frames of the actual recorded trajectory or only the cells that are present from the first to the last frames are considered (for MaxDist and FRDO).

The trajectory is generally smoother when generated by the automated method than by the manual method: the standard deviation of cell displacement is lower at each time step. On the other hand, the automated tracking takes into account the overall cell shape and thus may not able to accurately detect the position of the nucleus. Changes in cell shapes may interfere with the detection of cell nucleus.

Despite these difficulties, the results of the comparison between the manual vs the automatic method are found to be consistent for the three criteria used for characterizing the trajectories (MS, MaxDist, FRDO). The automated tracking was judged reliable and was therefore used to perform the analysis of the whole dataset. It thus allowed processing a large amount of data which is in turn expected to help reducing the influence of the tracking errors and the inherent variability of these analyses in order to extract general trends. 
A large part of this study was devoted to an experimental and quantitative analysis of melanocyte migration in vitro and in relation with cell-matrix interactions. In recent years, particular effort has been made to understand the molecular nature of extracellular matrices (ECM). These matrices are chemically complex structures that interact with cell membrane by a number of mechanisms. Interactions between cell surface receptors (mostly integrins) and various molecules of the ECM underlie a wide variety of processes including adhesion, spreading and motility. Adhesive noncollagenous glycoproteins, such as vitronectin, laminin and fibronectin (FN), serve pivotal roles in basement membrane and stromal matrices, respectively. These proteins participate in establishing the architecture of the ECM, which not only provides a substrate for cellular adhesion but also affects the cellular phenotype. The effect on the phenotype ranges from adhesion and motility to spreading, growth and differentiation.

Regardless of the ability of cells to migrate on a given substratum, their speed depends on several variables such as ligand levels, integrin levels, and integrin-ligand interactions [26]. We have chosen to focus particularly on the processes of spreading and migration by considering FN, which served as a prototype of the ECM glycoproteins. Density gradients of FN bound substratum can directly promote migration of cells in vitro (review in [24]). Accordingly, our results obtained in a wild-type melanocytes cell line show that cell motility increased significantly at a high concentration of FN coating. It has been determined that migration was relatively slow with low concentrations of substratum-bound or low integrin expression [17].

We also investigated the effect of fibronectin concentration on cell shape and its consequence on migration. After ECM attachment, integrin-dependent signaling initiates several events that finally results in changes in cell shape, or spreading, from a round morphology to that of an irregular flattened shape. Melanocytes, derived from neural crest cells, are usually round or oval, but fusiform and dendritic shapes have also been recognized [3]. Dendritic shapes have also been observed in melanocytes of the wild-type cell line. In our experiments, dendritic melanocytes were prone to form aggregates and showed no displacement when they were cultured on plastic without any coating. However, the morphology altered and they migrated with preferential direction when cultured with high fibronectin coating. This type of migration supposes that the cells might be activated by an external factor either chemical, (chemoattractant) or mechanical (heterogeneous rigidity of the environment) and responded by changing their morphology and motion pattern [30].

Another important point of this study is also to provide an analysis of the cross interactions between the effects of expression of mutant $\beta$-catenin and FN bound to the substratum on adhesion, spreading, migration and division. $\beta$-catenin is a multi-functional protein involved in cell-cell adhesion, intracellular signalling and gene transcription. It is expressed throughout the development of the melanocyte cell lineage, where the cell-cell adhesion function of $\beta$-catenin is mediated by the large cadherin cell adhesion molecule family [21]. Moreover, the production of cadherins and catenins is very plastic and this plasticity is very important in fundamental cellular mechanisms associated with the transformation status of the cells [18].

Interestingly, two types of trajectories as a function of cell shape were observed in the melanocytes of the bcat* cell line cultured on high concentrations of coating FN $(40 \mu \mathrm{g} / \mathrm{ml})$. The first type was characterized by high trajectory amplitude and final displacement linked to fusiform or dendrite shapes as observed in the wild type melanocytes. The second type presented no or very small 
displacement associated with a large irregular flattened shape. The relationship between migration and adhesion is not so simple and many details are still poorly understood. Migration is relatively slow when attachment to the substrate is low, because weakly attached cells do not generate enough traction to move significantly. However, cells may also display impaired motility on strongly adhesive surfaces. They may be well-spread and immobilized, presumably due to the inability to cycle between adherent and non adherent states [17, 26]. DiMilla et al. [13] have demonstrated that migration speed depends in biphasic manners on attachment strength. They conclude that a maximal migration was obtained at intermediate level of cell-substratum adhesiveness.

$\beta$-catenin have also an additional role in controlling the expansion of cells which change their fate. Mooney et al. [25] have demonstrated in primary hepatocytes that polymerization of either microfilaments or microtubules are not directly responsible for the spreading and flattening of the cell body. The significance of cell shape changes may relate to associated changes in cytoskeletal organization and structure, rather than general alterations in cytoskeletal filament mass. $\beta$-catenin is also able to regulate the transcription of genes involved in proliferation. Under these specific conditions, we showed that expression of a stable mutant of bcat* provokes a statistically significant increase in rate of cell division. In our experiments on melanocytes in vitro, increases in cell density were not accompanied by significant decreases in the mean speed of the cell population, as is often reported [22].

In conclusion, this study provided several results which allow better understanding of melanocyte migration. Moreover, the quantitative analysis provides a complex picture of interdependent action of molecular ( $\beta$-catenin over-expression) and environmental (adhesion to the substratum, cell density) conditions on motility, migration and cell shape.

We have demonstrated the accuracy of an automated system for tracking migration of unlabeled cells using phase-contrast microscopy. The labour intensiveness of cell migration analysis has been a major obstacle for research on cell migration. Different treatments (whether drug, matrix or gene based) often result in small changes in cell migration, seen in a background of high variability within each treatment. Our mean-shift algorithm based tracking is a significant advance in migration research, and it is able to be applied to other cell systems. The automated method allows processing large numbers of data which is interesting for biological experimental studies and for acquiring the knowledge necessary for further modeling works.

We have shown interactions (that have not yet been studied) between expression levels of $\beta$ catenin and effects of fibronectin bound to substratum, but in the future, the experimental protocol should include more intermediate concentrations of fibronectin to determine the concentration allowing the maximal migration.

As we have reported, increases in cell density were not accompanied by significant decreases in cell displacements. It is possible that the seeding density in our experiments is too low to produce sufficient levels of crowding to affect cell migration. Further experiments with successively higher seeding rates could determine what cell density affects migration in melanocytes in vitro, and whether cell matrix is an important determinant of response to cell crowding.

Our results on effects of fibronectin and on specificities of bcat* cell line on cell migration should be further investigated using 3D in vitro assays, as several studies have demonstrated the 
importance of considering the 3D cell displacement to get reliable results $[11,16]$.

\section{Acknowledgements}

We thank Fabrice Cordelières from the imaging facility of Institut Curie. S. Gallagher was supported by an Institut Curie fellowship. M. Kumasaka was supported by Ligue de 1' Oise and Ligue nationale contre le cancer fellowships. This work was supported by the Ligue Nationale Contre le Cancer (Equipe labellise), INCa, cancropole IdF. The internship of G. Letort was supported by ECP.

\section{References}

[1] M. Abal, M. Piel, V. Bouckson-Castaing, M. Mogensen, J.-B. Sibarita, M. Bornens. Microtubule release from the centrosome in migrating cells . The Journal of Cell Biology, 159 (2002), 731-737.

[2] S. Allard, P. Adin, L. Goudard, N. di Clemente, N. Josso, M.-C. Orgebin-Crist, J.-Y. Picard, F. Xavier. Molecular mechanisms of hormone-mediated Müllerian duct regression: Involment of $\beta$-catenin. Development, 127 (2000), 3349-360.

[3] K.J. Busam, C. Charles, G. Lee, A.C. Halpern. Morphologic Features of Melanocytes, Pigmented Keratinocytes, and Melanophages by In Vivo Confocal Scanning Laser Microscopy. Mod. Pathol., 14 (2001), 862-868.

[4] Y. Cheng. Mean shift, mode seeking, and clustering. IEEE Trans. Pattern Anal. Mach. Intell., 17 (1995), No. 8, 790-799.

[5] A. Dammermann, A. Desai, K. Oegema. The minus end in sight. Current Biology, 13 (2003), 614-624.

[6] O. Debeir, I. Camby, R. Kiss, P. Van Ham, C. Decaescker. A Model-Based Approach for Automated In Vitro Cell Tracking and Chemotaxis Analyses. Cytometry, 60A (2004), 29-40.

[7] O. Debeir, P. Van Ham, R. Kiss, C. Decaestecker. Tracking of Migrating Cells under Phasecontrast Video Microscopy with Combined Mean-Shift Processes. IEEE Transaction on Medical Imaging., 24 (2005), No. 6, 697-711.

[8] O. Debeir, V. Mégalizzi, N. Warzée, R. Kiss, C. Decaestecker. Videomicroscopic extraction of specific information on cell proliferation and migration in vitro. Experim. Cell Res., 314 (2008), 2985-2998. 
[9] V. Delmas, F. Beermann, S. Martinozzi, S. Carreira, J. Ackermann, M. Kumasaka, L. Denat, J. Goodall, F. Luciani, A. Viros, N. Demirkan, B.C. Bastian, C.R. Goding, L. Larue. $\beta$ catenin induces immortalisation of melanocytes by suppressing p16INK4a expression and co-operates with N-Ras in melanoma development. Genes \& Dev., 21 (2007), 2923-2935.

[10] N. Desban, J-L. Duband. Avian neural crest cell migration on laminin: interaction of the al1 integrin with distinct laminin-1 domains mediates different adhesive responses. J. Cell Sci. 110 (1997), 2729-2744.

[11] C. Decaestecker, O. Debeir, P. Van Ham, R. Kiss. Can anti-migratory drugs be screened in vitro ? A review of $2 D$ and $3 D$ assays for the quantitative analysis of cell migration. Inc. Med. Res. Rev., 27 (2007), No. 2, 149-176.

[12] R Development Core Team. R: A Language and Environment for Statistical Computing. R Foundation for Statistical Computing, Vienna, Austria., (2008), http://www.R-project.org

[13] P.A. DiMilla, J.A. Stone, J.A. Quinn, S.M. Albelda, D.A. Lauffenburger. Maximal Migration of Human Smooth Muscle Cells on Fibronectin and Type IV Collagen Occurs at an Intermediate Attachment Strength. J Cell Biol., 122 (1993), 729-37

[14] A.J. Hand, T. Sun, D.C. Barber, D.R. Hose, S. Macneil. Automated tracking of migrating cells in phase-contrast video microscopy sequences using image registration. J. Microscopy., 234 (2009), 62-79.

[15] N. Hamilton. Quantification and its Applications in Fluorescent Microscopy Imaging. Traffic, 10 (2009), 951961.

[16] S. Hazgui, N. Bonnet, J. Cutrona, B. Nawrocki-Raby, M. Polette, L. Chouchane, P. Birembaut, J-M. Zahm. 3D culture model and computer assisted videomicroscopy to analyze migratory behavior of noninvasive and invasive bronchial epithelial cells. Am. J. Physiol. Cell Physiol., 289 (2005), 1547-1552.

[17] S.P. Holly, M.K. Larson, L.V. Parise. Minireview. Multiple Roles of Integrins in Cell Motility. Exp. Cell Res., 261 (2000), 69-74.

[18] A. Jouneau, Y.-Q. YU, M. Pasdar, L. Larue. Plasticity of Cadherin-Catenin Expression in the Melanocyte Lineage. Pigment Cell Res. 13 (2000), No.4, 260-272.

[19] E. Knust, W.B. Huttner. Cell polarity from cell division. Dev. Cell., 12 (2007), 664-666.

[20] L. Larue, N. Dougherty, S. Porter, B. Mintz. Spontaneous malignant transformation of melanocytes explanted from Wf/Wf mice with a Kit kinase-domain mutation. Proc. Natl. Acad. Sci. USA., 89 (1992), 7816-7820.

[21] L. Larue, M. Kumasaka, C.R. Goding. Beta-catenin in the melanocyte lineage. Pigment Cell R., 16 (2003), 312-317. 
[22] Y. Lee, L.V. McIntire, K. Zygourakis. Analysis of endothelial cell locomotion: Differential effects of motility and contact inhibition. Biotechnol Bioeng. 43 (1994), 622-634.

[23] T.C. Mayer. The migratory pathway of neural crest cells into the skin of mouse embryos. Dev. Biol., 34 (1973), 39-46.

[24] J. McCarthy, E.A. Turley. Effects of Extracellular Matrix Components on Cell Locomotion. Critical Reviews in Oral Biology and Medecine., 4 (1993), 619-637.

[25] D.J. Mooney, R. Langer, D.E. Ingber. Cytoskeletal filament assembly and the control of cell spreading and function by extracellular matrix. J. Cell Sci., 108 (1995), 2311-2320.

[26] S.P. Palecek, J.C. Loftus, M.H. Ginsberg, D.A. Lauffenburger, A.F. Horwitz. Integrin-ligand binding properties govern cell migration speed through cell-substratum adhesiveness. Nature, 385 (1997), 537-540.

[27] A.J. Ridley, M.A. Schwartz, K. Burridge, R.A. Firtel, M.H. Ginsberg, G. Borisy, J.T. Parsons, A.R. Horwitz. Cell migration: integrating signals from front to back. Science., 302 (2003), 1704-1709.

[28] C. Rosello, P. Ballet, E. Planus, P. Tracqui. Model driven quantification of individual and collective cell migration. Acta Biotheoretica, 52 (2004), 343-363.

[29] G. Scott, S. Leopardi, S. Printup, B.C. Madden. Filopodia are conduits for melanosome transfer to keratinocytes. J. Cell Sci., 115 (2002), 1441-1451.

[30] A. Stéphanou, E. Mylona, M. Chaplain, P. Tracqui. A computational model of cell migration coupling the growth of focal adhesions with oscillatory cell protusions. Journal of Theor. Biol., 253 (2008), 701-716.

[31] M. Tawk, C. Araya, D.A. Lyons, A.M. Reugels, G.C. Girdler, P.R. Bayley, D.R. Hyde, M. Tada, J.D. Clarke. A mirror-symmetric cell division that orchestrates neuroepithelial morphogenesis. Nature, 446 (2007), No. 7137, 797-800.

[32] M. Théry, V. Racine, A. Pépin, M. Piel, Y. Chen, J.-B. Sibarita, M. Bornens. The extracellular matrix guides the orientation of the cell division axis. Nature Cell Biology, 7 (2005), No. 10, 947-953.

[33] A.L. Wilkie, S.A. Jordan, I.J. Jackson. Neural crest progenitors of the melanocyte lineage: coat colour patterns revisited. Development, 129 (2002), 3349-57. 